\title{
Thomson scattering analysis of large scale fluctuations in the ASDEX Upgrade edge
}

\author{
B Kurzan, L D Horton, H Murmann, J Neuhauser, W Suttrop, \\ and ASDEX Upgrade Team
}

\section{Max-Planck-Institut für Plasmaphysik, EURATOM Association, Boltzmannstr. 2, 85748 Garching, Germany}

Email: Bernd.Kurzan@ipp.mpg.de

\begin{abstract}
Large scale fluctuations in between edge localized modes (ELMs) are the main source for the scatter in plasma edge $\mathrm{H}$ mode profiles of electron density and temperature, as measured by high precision, high resolution Thomson scattering. These large scale fluctuations are also observed with electron cyclotron emission. They are quantitatively analysed by $2 \mathrm{D}$ poloidal snapshots of electron density and temperature, based on a $5 \times 10$ matrix of scattering volumes provided by the Thomson scattering system. Fluctuations with a quasi-periodic structure are found in a $2 \mathrm{D}$ snapshot with a frequency of about $61 \%$. When interpreted as field-aligned helical structures toroidal quasi-mode numbers of 6 to 48 are found. The amplitudes of the fluctuations decrease with increasing quasi-mode number and edge profile gradient lengths. The amplitudes of the large scale structures in the steep gradient region are anticorrelated with the divertor $D_{\alpha}$-intensity. The particle loss during an ELM is at least to a significant fraction due to the electron density 'blobs' observed in the SOL. The large scale fluctuations also perturb the measurement of $1 \mathrm{D}$ radial profiles. In the middle of the steep gradient region the perturbations are symmetric, but asymmetric both further inside (more minima), and further outside (more maxima).
\end{abstract}




\section{Introduction}

In divertor tokamaks the plasma edge is crucial for the performance of the core plasma. When the plasma changes from a low $(\mathrm{L})$ to a high $(\mathrm{H})$ confinement mode [1], an edge barrier builds up, which is characterized by increased pressure gradients, poloidal rotation and velocity shear at the plasma edge.

The large pressure gradients in $\mathrm{H}$ mode plasmas give rise to large scale magnetohydrodynamic (MHD) modes in the confinement zone inside the separatrix. One such strong instability is the type-I edge localized mode (ELM) [2] exhausting heat and particles in short $(\approx 100 \mu s)$ bursts at a repetition rate of a few 10 s or 100 s of Hz. On ASDEX Upgrade the fluctuation structures during and in between type-I ELMs [3] were investigated near the separatrix and in the scrape-off layer (SOL) by 2D Thomson scattering [4], on first wall and limiter structures by 2D thermography [5] [6], in the far SOL by Langmuir probes [7] [8] and by magnetic probes [9]. The mode dynamics at the plasma edge have been investigated by Electron Cyclotron Emission (ECE) [9] and reflectometry [10] [11]. Precursors to ELMs were found on ASDEX Upgrade and other experiments (see [9] and [12] and references therein).

Modes, which are localized at the edge but exist in inter-ELM phases, have been observed in several machines: At JET the washboard modes [13][14] and at Alcator C-Mod the quasi-coherent mode [15] exist. Large scale modes existing at the plasma edge also in inter-ELM phases at ASDEX Upgrade have been found [3], but have not yet been characterized further and in great detail.

In this paper, these large scale fluctuations of electron density and temperature in the steep edge gradient region and the SOL in inter-ELM phases and during the ELM are investigated quantitatively using mainly Thomson scattering data.

The paper is organized as follows: In section 2 the experimental setup including the discharge parameters and the diagnostics are described. In section 3 the results are presented, followed by a discussion of the results in section 4 .

\section{$2 \quad$ Experimental setup}

The vertical Thomson scattering diagnostic on ASDEX Upgrade was designed and built to allow great flexibility in the spatial locations and time schemes for the measurement of electron density and temperature profiles while still providing a large signal-to-noise ratio necessary for high accuracy [16]. The light source presently consists of 5 vertically launched radially separated Nd-YAG laser beams. The scattered light is imaged directly through air onto 16 polychromator channels on the low field side of the torus, thus defining 16 vertically separated scattering volumes in the plasma. The whole diagnostic can be shifted to measure either plasma core, or edge profiles. At the plasma edge only the upper 10 spatial channels detect Thomson scattered light (see figure 1). A single scattering volume has a length of $25 \mathrm{~mm}$ in the vertical direction and a diameter of about $1.5 \mathrm{~mm}$, which is the laser beam's diameter. The distance between the scattering volume centres in the vertical direction is $60 \mathrm{~mm}$, and the radial distance between adjacent lasers is $2.7 \mathrm{~mm}$. At the plasma 


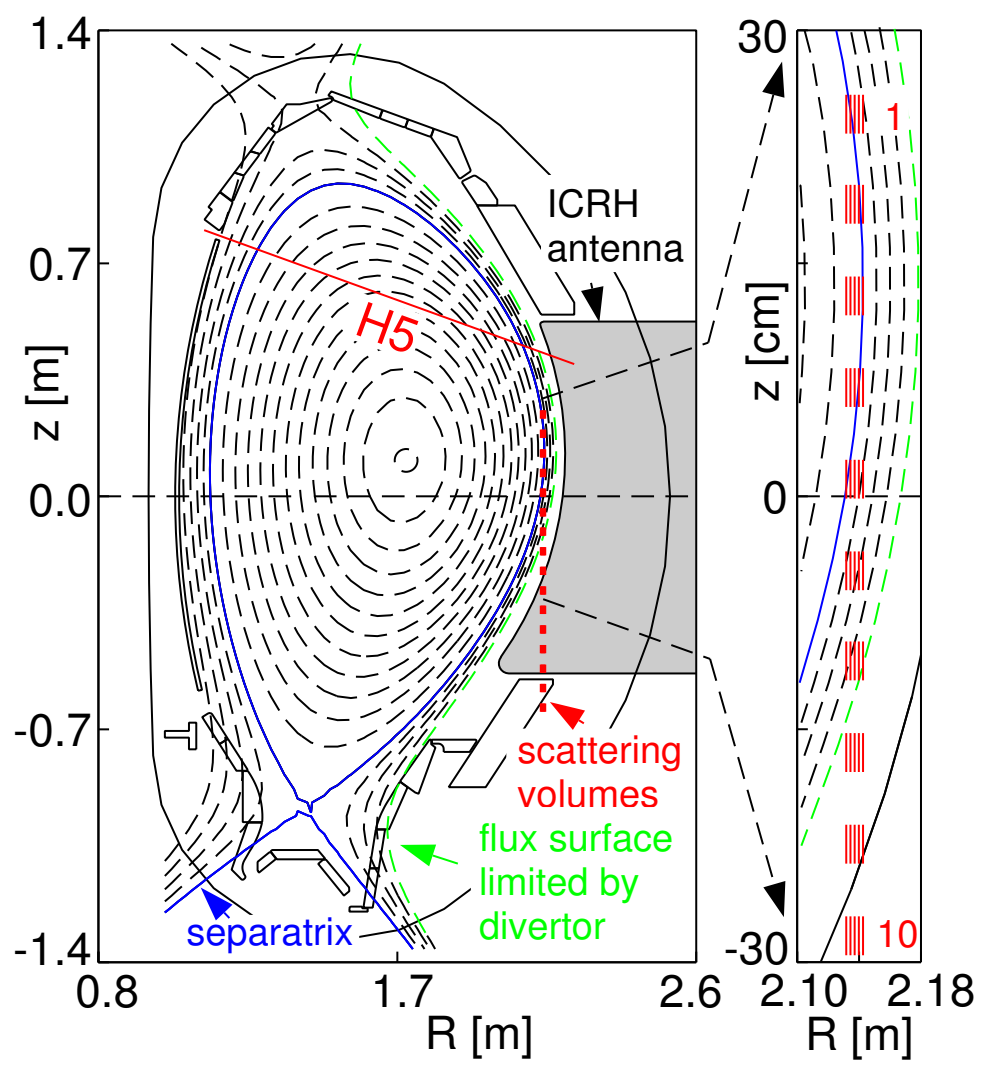

Figure 1: Poloidal cross section of the ASDEX Upgrade vessel with the DCNinterferometer line integral $H 5$ and the scattering volumes of the Thomson scattering diagnostic. With the 5 radially separated lasers a $2 D(R, z)$-matrix of $(5 \times 10)$ scattering volumes is formed at the plasma edge (see zoomed-in detail).

edge this forms a vertically stretched $(\mathrm{R}, \mathrm{z})$ matrix of $5 \times 10$ scattering volumes (see zoom in figure 1). This $2 \mathrm{D}$ resolution capability of the Thomson scattering diagnostic is available when the lasers are fired consecutively in time. With the use of ultrafast transient recorders for data acquisition, it is possible to operate the lasers in burst mode where the time delay between the lasers is as small as $500 \mathrm{~ns}$. This minimum delay time was used for the five lasers which were available for the experiments described in this paper. They were fired within $2 \mu \mathrm{s}$, defining the 'exposure time' for the measured 2D snapshots of electron density and temperature. Such 2D snapshots are obtained every $50 \mathrm{~ms}$, which is the repetition time of the lasers.

Recently the accuracy of the evaluated data has been improved considerably, even beyond the status that is described in [17]. The statistical errors of the measured electron densities and temperatures, which are due to photon statistics, and the noise of the detector, the amplifier, and the plasma light, are estimated in the data evaluation process. The resulting relative statistical errors for electron densities $n_{e} \geq 5 \times 10^{18} \mathrm{~m}^{-3}$ are now $\delta n_{e, s t a t} / n_{e} \leq 10 \%$ and $\delta T_{e, s t a t} / T_{e} \leq 15 \%$. There are examples (\#18397/8) where at some centimetres inside the profile pedestal shoulder the plasma fluctuations are so small that the scatter of the electron densities is indeed 
within the estimated statistical error of measurement of in this case $\delta n_{e, s t a t} / n_{e} \approx 3 \%$.

Although the sources of all the still existing systematic errors are not yet known, their relative size can be estimated from the channel-to-channel variation of assumedto-be smooth profiles. The estimated relative systematic errors are $\delta n_{e, s y s} / n_{e} \approx 7 \%$ and $\delta T_{e, s y s} / T_{e} \approx 7 \%$.

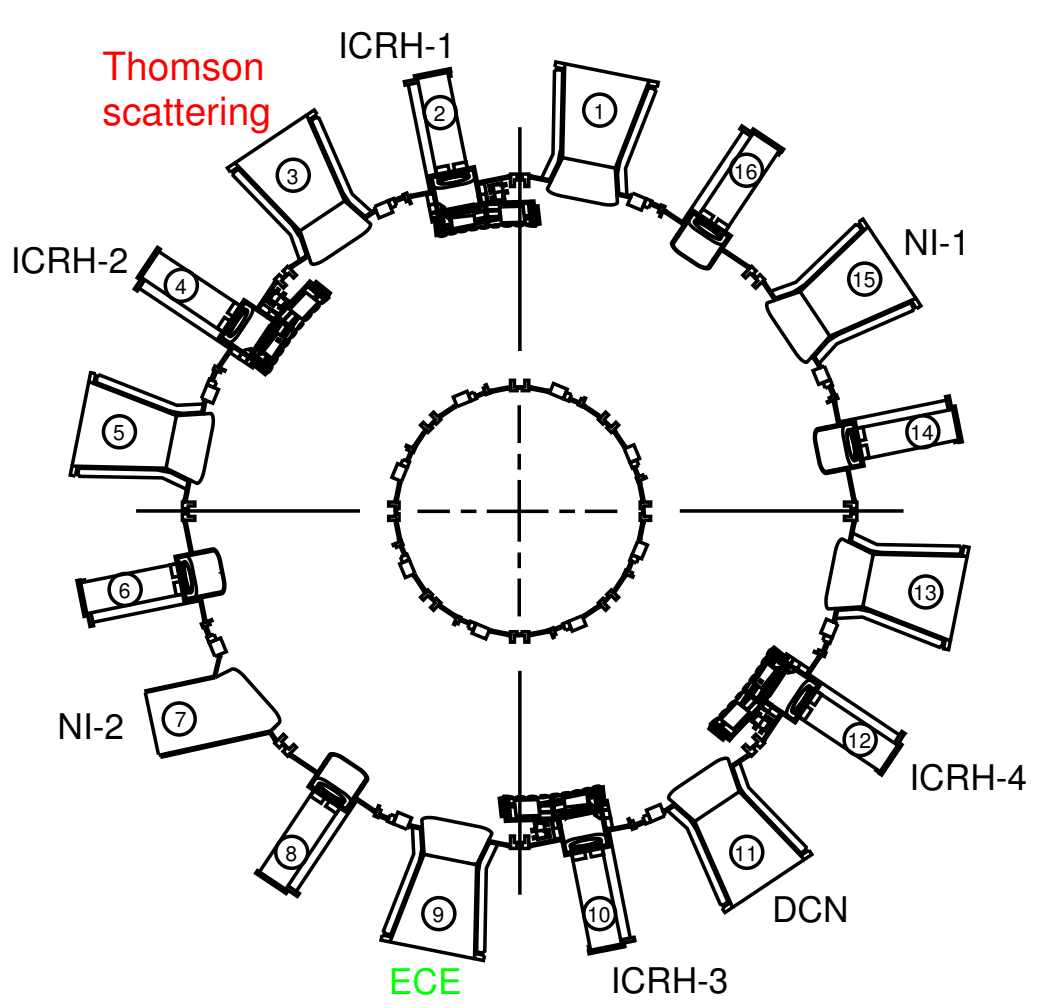

Figure 2: Horizontal cut of the ASDEX Upgrade torus with the positions of Thomson scattering, ECE, and the DCN-interferometers.

In this paper the Thomson scattering data are compared to ECE data (sampling rate $31.25 \mathrm{kHz}$ ) and a selected DCN-interferometer line integral, $\mathrm{H} 5$ (see figure 1). These diagnostics are distributed along the toroidal direction (see figure 2).

The parameters of the discharges which are investigated in this paper are summarized in table 1 . The data set includes variations in line-density $\bar{n}_{e}$, heating powers $\left(P_{N B I}, P_{I C R H}\right)$, and equilibrium parameters $\left(q_{95}, \kappa, \delta_{u}\right)$. In this paper, plasma plateau phases with different parameters are studied within a single discharge or a group of similar discharges.

\section{Results}

Before characterizing the large scale fluctuations in the plasma edge, the general behaviour of the $1 \mathrm{D}$ radial electron density and temperature profiles in inter-ELM and ELM phases is presented. 
Table 1: Discharge parameters: toroidal magnetic field $B_{t}$, plasma current $I_{p}$, safety factor $q_{95}$, line density $\bar{n}_{e}$, power by neutral beam co-injection $P_{N B I}$, power by ion cyclotron resonance heating $P_{I C R H}$, elongation $\kappa$, upper triangularity $\delta_{u}$, lower triangularity $\delta_{l}$.

\begin{tabular}{l|llll}
\hline shot numbers & 19807 & $20414-20420$ & $19046-19047$ & 20647 \\
\hline Confinement mode & $\mathrm{H}$ & $\mathrm{H}$ & $\mathrm{H}$ & $\mathrm{L}$ \\
$B_{t}[T]$ & -2.5 & -2.7 & -3.0 & -2.5 \\
$I_{p}[M A]$ & 1.00 & 1.00 & 0.80 & 0.80 \\
$q_{95}$ & 4.4 & $5.5 \pm 0.1$ & 7.5 & 5.4 \\
$\bar{n}_{e}\left[10^{19} \mathrm{~m}^{-3}\right]$ & 8.7 & $6.4-8.4$ & $4.7-5.5$ & 6.0 \\
$P_{N B I}[M W]$ & 7.5 & $5-10$ & 7.5 & 1.4 \\
$P_{I C R H}[M W]$ & 0 & $1.3-1.9$ & 0 & 0 \\
$\kappa$ & 1.78 & $1.80 \pm 0.04$ & 1.83 & 1.72 \\
$\delta_{u}$ & 0.17 & $0.155 \pm 0.025$ & 0.24 & -0.055 \\
$\delta_{l}$ & 0.40 & 0.38 & 0.46 & 0.35 \\
\hline
\end{tabular}

\subsection{D radial $H$ mode profiles of electron density and tem- perature}

In $\mathrm{H}$ mode two extreme types of radial edge profiles of electron density and temperature are observed: Profiles with steep gradients are found before and after an ELM, and flatter profiles exist during the ELM due to the erosion of the pedestal. This was already investigated by reflectometry [10].

The time averaged 1D radial electron density and temperature Thomson profiles are plotted in figure 3 for phases before, during, and after the ELM. Such 1D radial profiles are obtained by mapping the measured electron density and temperature data along the poloidal flux surfaces to the equatorial plane of the plasma on the low field side. The radial coordinate is the major radius $R$ minus the outermost position of the separatrix $R_{\text {out }}$. The plasma equilibria were obtained from the equilibrium code CLISTE [18]. The error in the radial position of the separatrix is $5 \mathrm{~mm}$.

The divertor $D_{\alpha}$-intensity reaches a maximum during the ELM at the time $t_{E L M}$. Directly after the maximum in the divertor $D_{\alpha}$-intensity, both electron density, and temperature profiles have the flattest gradients in the ELM cycle. The electron density and temperature profiles are again as steep as before the ELM about $3 \mathrm{~ms}$ after the maximum in the divertor $D_{\alpha}$-intensity. These steep edge profiles are maintained until the next ELM, which occurs after about $10 \mathrm{~ms}$.

\subsection{Identification of inter-ELM fluctuations on the ECE di- agnostic}

Large inter-ELM fluctuations, as observed by 2D Thomson scattering, should be visible also on the ECE diagnostic, if their spectra overlap with the video frequency range $(0-15 k H z$ on the system at ASDEX Upgrade). In order to check this an example with large structures is chosen. 

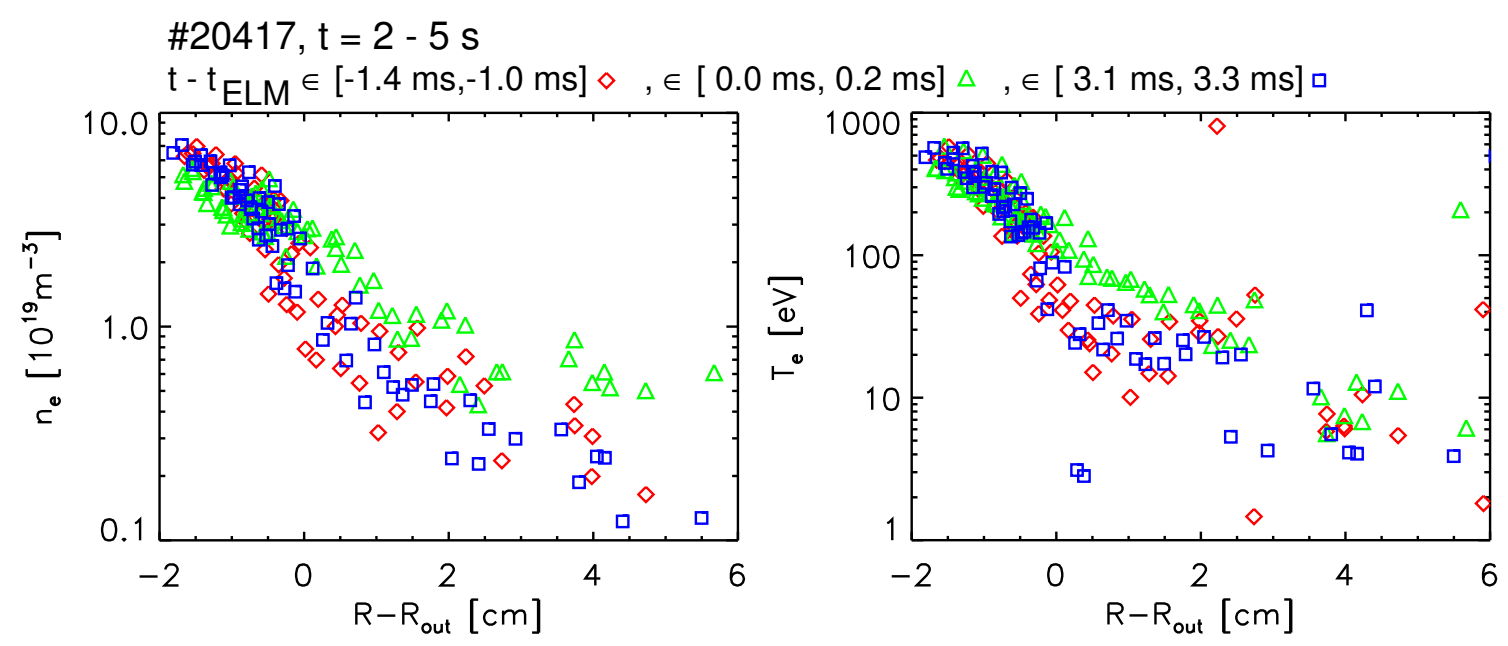

Figure 3: 1D radial electron density and temperature profiles before, during and after ELMs in the plateau phase, $t=2-5 s$, of \#20417. Before the ELM (red diamond symbols), the profiles are steep. After the maximum in the divertor $D_{\alpha}$-intensity, (green triangle symbols), the profiles have the flattest gradients in the ELM cycle. The profiles are steep again about $3 \mathrm{~ms}$ after the maximum in $D_{\alpha}$ (blue square symbols).

In the following the $2 \mathrm{D}$ snapshots of electron density and temperature, are visualized in contour plots. The radial coordinate is the major radius $R$ minus the outermost radial position $R_{\text {out }}$ of the separatrix on the low field side. In the $2 \mathrm{D}$ plots of electron density and temperature contour lines of the poloidal flux are overlaid.

To make fluctuation structures of the electron density and temperature clearly visible, an averaged snapshot is subtracted from each single snapshot, resulting in the relative electron densities $\Delta n_{e}$ and temperatures $\Delta T_{e}$. The fluctuation structures change completely from one single snapshot to the other. When adding up several snapshots the maxima and minima in the single snapshots are partially compensated and the structures visible in the averaged snapshot have quite small amplitudes. Averaging over 20 snapshots is sufficient to reduce the relative fluctuation amplitudes in the averaged snapshots to about $5 \%$. Such an analysis can only be done in plateau phases of the discharge, where the plasma parameters are constant.

A typical example for the large scale fluctuations observed by $2 \mathrm{D}$ Thomson scattering in the steep gradient region is shown in figure 4(a) (a more extreme case can be found in [3]). In this case two maxima and one minimum in the electron density and temperature are visible in the relative amplitudes $\Delta n_{e}$ and $\Delta T_{e}$. These fluctuations should stay constant for times much greater than the 'exposure' time of the snapshots, of $2 \mu \mathrm{s}$. Otherwise the structure would be smeared out and would become invisible to the Thomson scattering diagnostic.

With the ECE diagnostic also signal spikes in inter-ELM phases are typically observed in three to five channels in the steep gradient region near the separatrix. In the example, which is shown in figure 4(b), positive, and negative spikes in the electron temperature are observed in the innermost and the outermost channel of a 

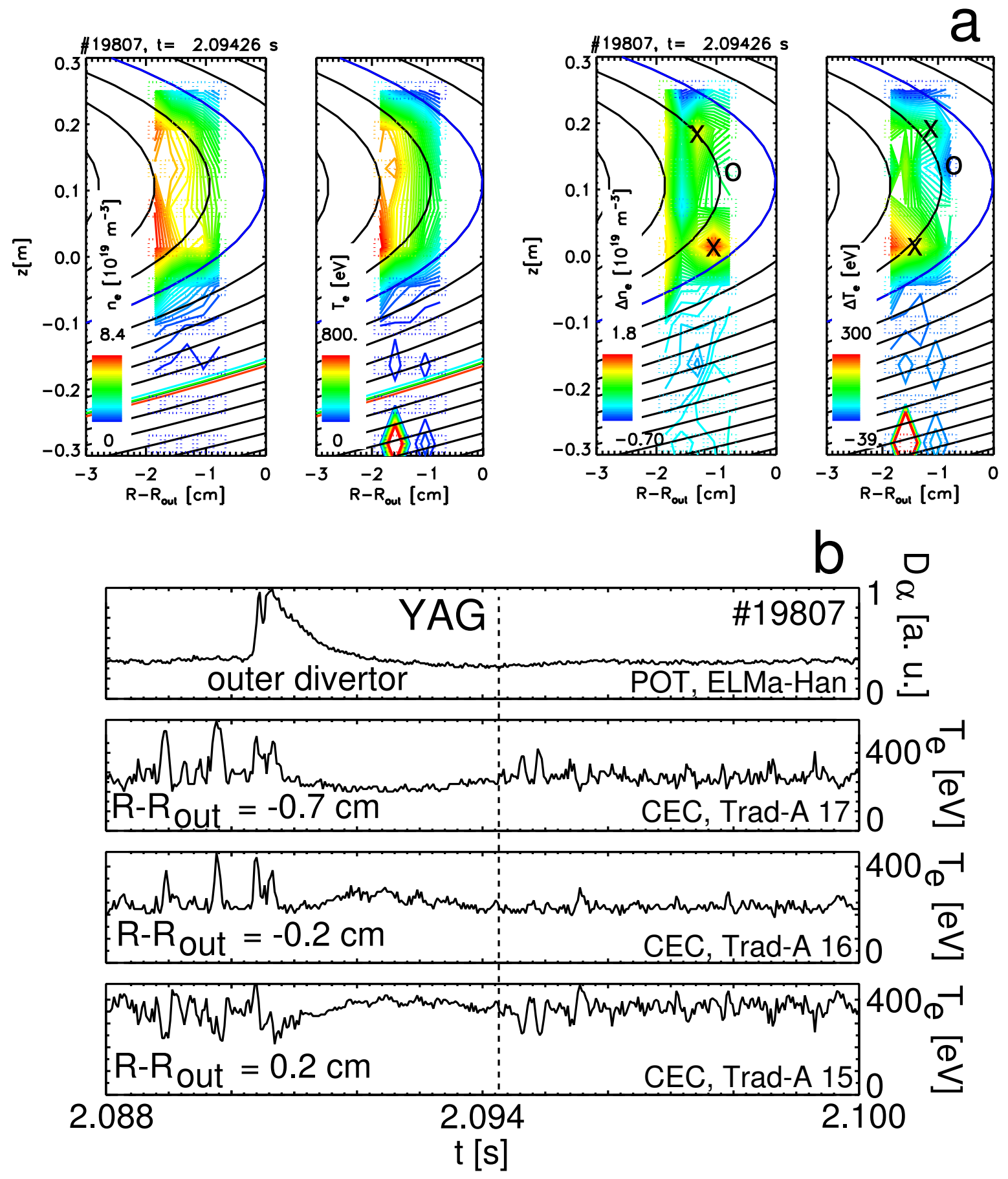

Figure 4: In the steep gradient region near the separatrix two maxima and one minimum, marked by the symbols ' $x$ ' and ' $o$ ' respectively, are visible in the relative electron density and temperature amplitudes $\Delta n_{e}$ and $\Delta T_{e}$ in the $2 D$ snapshots (a). In the steep gradient region near the separatrix a spatial structure is observed in the electron temperature in three adjacent ECE channels. The inner- and outermost channels show positive, and negative spikes respectively, before an ELM, and again $3.5 \mathrm{~ms}$ after the maximum in the $D_{\alpha}$-intensity (b). 
group of three adjacent channels. The duration of these spikes is about $100 \mu s$ and the relative amplitudes are $\Delta T_{e, \text { rad }} / T_{e, \text { rad }} \leq 0.5$.

Due to present limitations in the sampling frequency of $31.25 \mathrm{kHz}$ the true duration of the signal spikes might be shorter and their amplitudes larger. One should notice that the absolute value of the electron radiation temperature $T_{e, \text { rad }}$ derived from ECE near the separatrix is typically larger than the electron temperature measured by Thomson scattering, $T_{e, r a d}>T_{e}$, and shows a parasitic maximum there. This effect is well known and attributed to a small non-thermal electron population at high electron temperature gradients and low electron density values resulting in insufficient optical thickness [19]. It has been argued [3] that the spikes might be due to island-type perturbations, but the (apparent) ECE temperature profile modulation in \#19807 would indicate a profile steepening rather than the expected flattening over an island. However, since there are also counter examples (\#19438) indeed exhibiting plateau formation, and in view of the optical thickness problem mentioned above, this question remains still open.

The spatial structure in the electron temperature, as observed by ECE, has a diameter of about $0.9 \mathrm{~cm}$ and is located in the steep gradient region near the separatrix. This is in agreement with the location of the fluctuations seen by $2 \mathrm{D}$ Thomson scattering (figure 4(a)). The signal spikes in ECE reach their maximum amplitude about $0.2 \mathrm{~ms}$ later, than they were observed by Thomson scattering, indicated by the line in figure 4(b). If the same fluctuation structure is observed by both diagnostics, then two things can be concluded: (a) The lifetime of this fluctuation structure must be at least $0.2 \mathrm{~ms}$. (b) This fluctuation structure apparently moves both in the poloidal, and/or toroidal direction, crossing the different locations of the two diagnostics (see figure 2) at different times. The signal spikes in ECE occur very often before an ELM, but after an ELM only with a delay of some milliseconds (see figure 4(b)). In the phases directly after an ELM, where these signal spikes do not occur, the edge gradient region is just steepening again, as can be seen in figure 4(b).

Phases of different mode activity can be found in the ECE signals (figure 4): The low activity after the ELM is followed by a phase of strong activity, lasting for about $5 \mathrm{~ms}$, after which the mode activity is low. About $3 \mathrm{~ms}$ before an ELM the mode activity rises again.

\subsection{Scaling of the large scale fluctuations with plasma pa- rameters}

Using the discharges \#20414 - 20 the large scale fluctuations in the steep gradient region can be investigated statistically. In these discharges mainly the line density $\bar{n}_{e}$ and heating powers $\left(P_{N B I}, P_{I C R H}\right)$ were varied from shot to shot (see table 1 ). The data are taken during the plateau phases of the discharges, where the plasma parameters were constant. A number of $5272 \mathrm{D}$ snapshots were investigated and 321 of them, or a fraction of $61 \%$, showed clearly quasi-periodic structures. From these 321 snapshots the properties of the large scale fluctuations are extracted and collected in a database. A typical example for inter-ELM fluctuation structures 

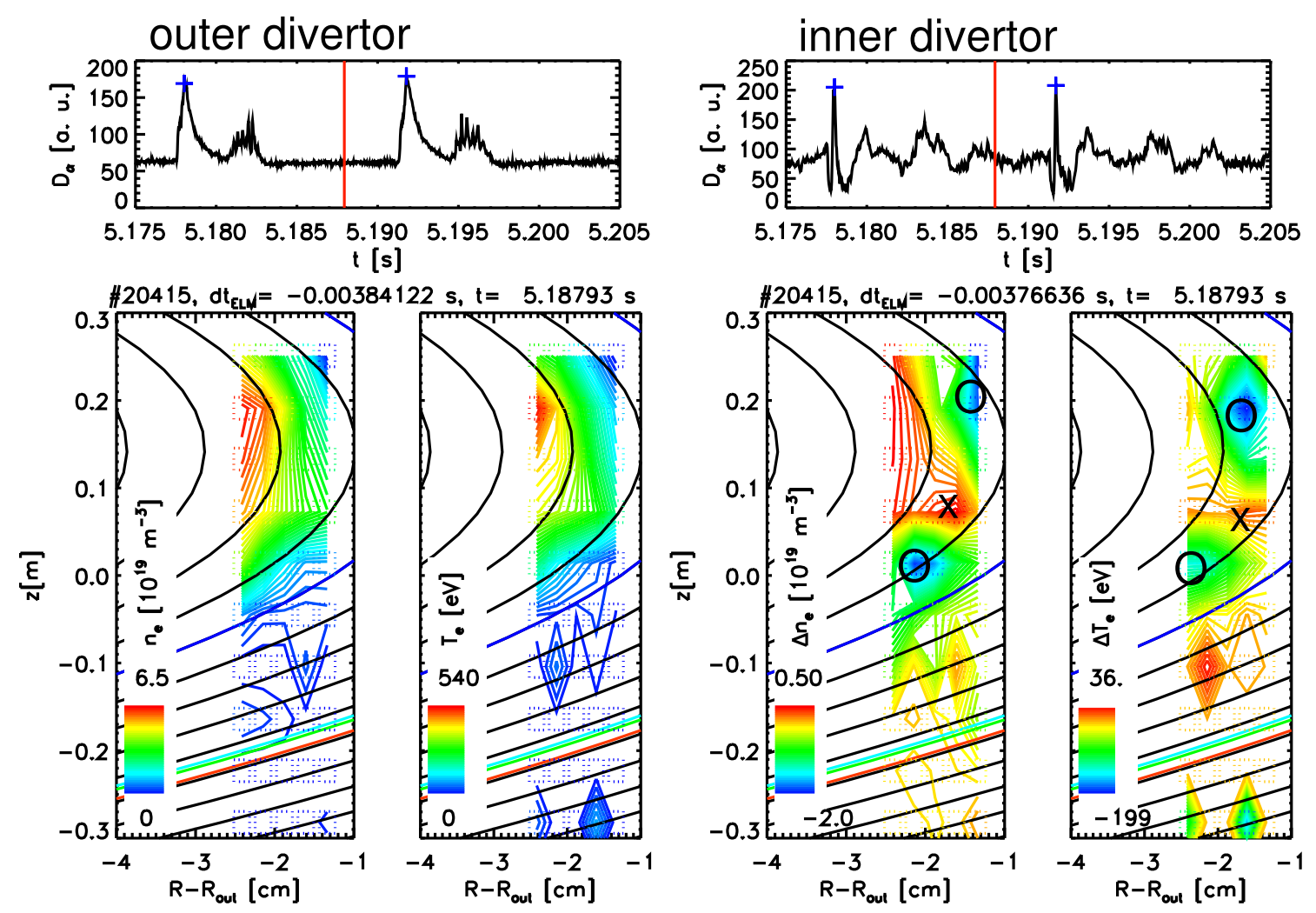

Figure 5: Inter-ELM fluctuation structures in electron density and temperature, $n_{e}$ and $T_{e}$, and in the relative amplitudes $\Delta n_{e}, \Delta T_{e}$. The maxima and minima are marked by the symbols ' $x$ ' and ' $o$ ' respectively. The toroidal quasi-mode number is here $n_{\text {tor }}=13.2$. The maxima in the $D_{\alpha}$-intensity during the ELMs at the times $t_{E L M}$ are indicated by the ' + ' symbols.

observed by 2D Thomson scattering is shown in figure 5 . For this group of discharges the divertor $D_{\alpha}$ radiation shows a double structure with the main $D_{\alpha}$ peak followed by a smaller, second maximum.

The structure of the large scale fluctuations in the 2D snapshots of electron density and temperature comes out clearly in the $2 \mathrm{D}$ plots of the relative amplitudes $\Delta n_{e}$ and $\Delta T_{e}$. In both the relative electron density and temperature, one maximum and two minima are observed at about $-1.3 \mathrm{~cm}$ inside the separatrix. The maxima and minima are at the same positions and in phase for both the electron density, and temperature. The absolute fluctuation amplitudes are $\tilde{n}_{e}=1.1 \times 10^{19} \mathrm{~m}^{-3}$ and $\tilde{T}_{e}=79 \mathrm{eV}$. When compared to the poloidally averaged background electron density $\left\langle n_{e}\right\rangle_{p o l}$ and temperature $\left\langle T_{e}\right\rangle_{p o l}$ relative fluctuation amplitudes of $\tilde{n}_{e} /\left\langle n_{e}\right\rangle_{p o l}=51 \%$ and $\tilde{T}_{e} /\left\langle T_{e}\right\rangle_{p o l}=73 \%$ are found in this case.

Although the maxima and minima in the electron density and temperature are at the same positions, the distances between the two minima and the maximum in the poloidal plane are not equal. This can be due to both the limited spatial resolution of the matrix of scattering volumes, and the transient nature of these fluctuations, which are not necessarily coherent modes. The poloidal wavelength of the fluctua- 
tions is estimated by determining the largest possible poloidal distance between two extrema of the fluctuation structure, and then dividing it by the number of enclosed periods (in general a multiple of $1 / 2$ ). With this method errors originating from the limited spatial resolution of the diagnostic are minimized. Taking into account the local pitch angle of the magnetic field and making the assumption that the mode structures are aligned to the magnetic field, a toroidal quasi-mode number can then be estimated [4]. For the discharges \#20414 - 20 toroidal mode numbers in the range $6 \leq n_{\text {tor }} \leq 55$ can be determined. The toroidal quasi-mode number for the fluctuation structure in figure 5 is $n_{\text {tor }}=13.2$.

The mode structures observed in the electron density profiles can be parametrized in the poloidal direction by $n_{e}\left(\theta^{*}\right)=\left\langle n_{e}\right\rangle_{p o l}+\tilde{n}_{e} \sin \left[n_{\text {tor }} q\left(\theta^{*}+\theta_{0}^{*}\right)\right]$ and analogously for the electron temperature. $\theta^{*}$ is the straight field line angle, $\theta_{0}^{*}$ is the poloidal phase of the electron density and temperature structures, which are in phase with each other, and $q$ is the safety factor.

To follow the variation of the fluctuation amplitudes with time relative to an ELM, the divertor $D_{\alpha}$-intensities and the fluctuation amplitudes of electron density and temperature in the steep gradient region, $\tilde{n}_{e}$ and $\tilde{T}_{e}$, taken from the database for the shots \#20414 - 20, are plotted versus the time $t-t_{E L M}$ in figure 6 . To emphasize the dynamics in the $D_{\alpha}$-light and to compare the $D_{\alpha}$-intensities for the shots \#20414 - 20, which have different $D_{\alpha}$-background light levels $D_{\alpha, \text { min }}$ between the ELMs, the relative intensities $\Delta D_{\alpha}=D_{\alpha}-D_{\alpha, \text { min }}$ are used here. When plotting all data points of the database (figure 6(a)), corresponding to fluctuation structures with quasi-mode numbers $n_{\text {tor }} \leq 48$, an average $D_{\alpha}$-light signal analogous to the non-averaged $D_{\alpha}$-signal in figure 5 (outer divertor) results: After the maximum in the $D_{\alpha}$-intensity during the ELM at the time $t_{E L M}$ another smaller maximum exists between $t-t_{E L M} \approx 2.5-5.7 \mathrm{~ms}$. The onset of the rise in the $D_{\alpha}$-light signal marking the beginning of the ELM is here about $0.4 \mathrm{~ms}$ before the maximum in the $D_{\alpha}$-intensity. The fluctuations in electron density and temperature show increased amplitudes before the ELM, where the $D_{\alpha}$-intensity is still low, and reduced amplitudes for a time interval of about $2.5 \mathrm{~ms}$ after the ELM onset, where the $D_{\alpha^{-}}$ intensity is high. For these phases the fluctuation amplitudes in the steep gradient region and the divertor $D_{\alpha}$-intensities are anti-correlated. In the phase where the amplitudes $\tilde{n}_{e}$ and $\tilde{T}_{e}$ of the fluctuations in electron density and temperature are reduced, the profiles of electron density and temperature are flatter than before the ELM and start to recover again (see figure 3). In the phase of the second maximum in the $D_{\alpha}$-intensity at $t-t_{E L M} \approx 2.5-5.7 \mathrm{~ms}$ the fluctuation amplitudes in the electron density seem to increase also (figure 6(a)). When plotting only the data belonging to the large scale structures with $n_{\text {tor }} \leq 10$ (figure $6(\mathrm{~b})$ ), which are mainly responsible for the dynamics in the amplitudes of electron density and temperature fluctuations, a rise in the fluctuation amplitudes is observed before the ELM and around $t-t_{E L M} \approx 2.5-5.7 \mathrm{~ms}$. The accordingly selected $D_{\alpha}$-intensities, however, no longer show the second maximum after the ELM (see figure 6(b)). This means again that large $D_{\alpha}$-intensities correlate with low amplitudes in the fluctuations of electron density and temperature.

To characterize these quasi-periodic fluctuations their spatial localizations, fluc- 

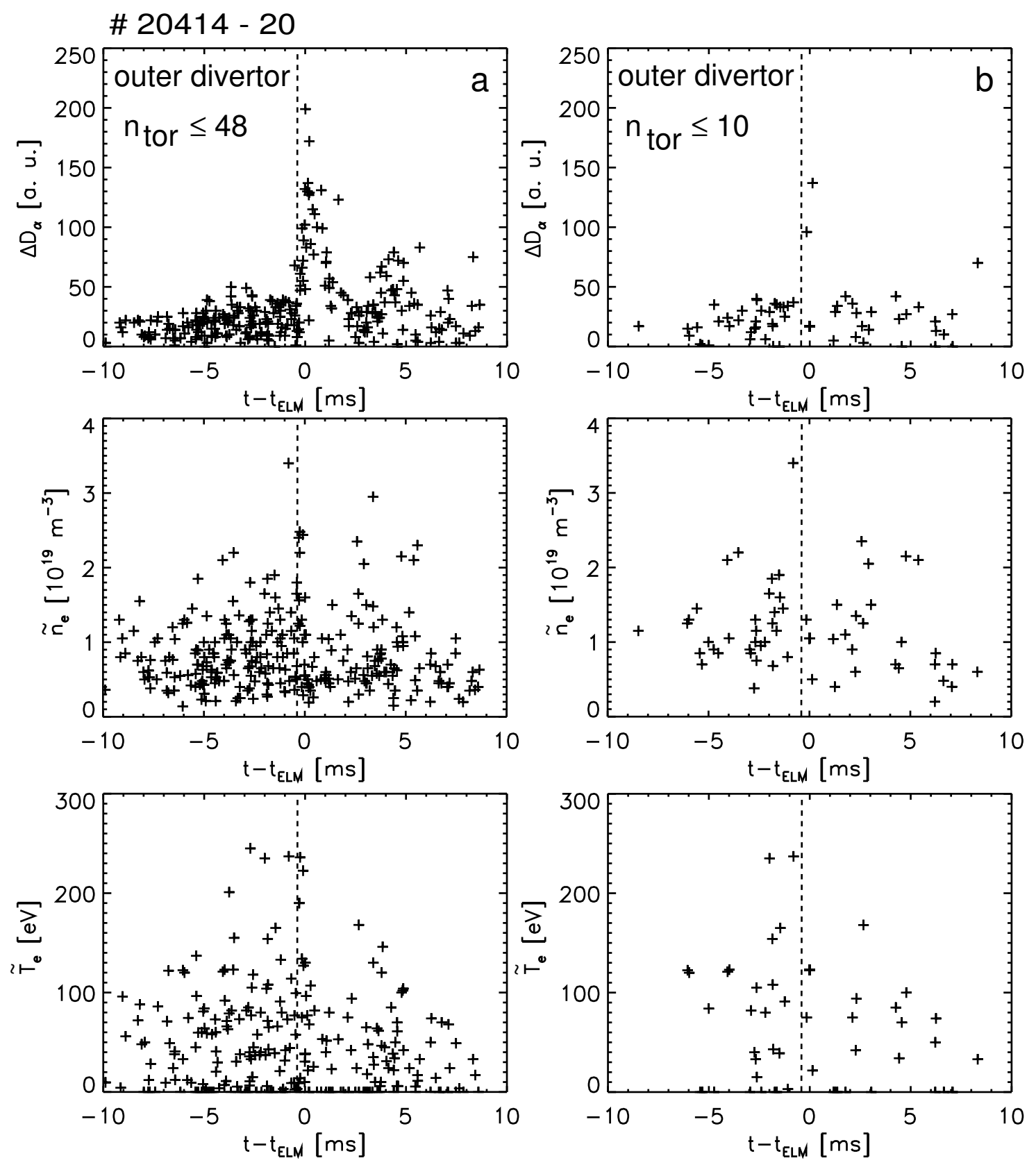

Figure 6: Statistical analysis (\#20414-20) of the time evolution of the relative $D_{\alpha}$ intensity, $\Delta D_{\alpha}$, and of the fluctuation amplitudes $\tilde{n}_{e}$ and $\tilde{T}_{e}$ for the mode numbers $n_{\text {tor }} \leq 48(a)$, and $n_{\text {tor }} \leq 10(b)$. The averaged $D_{\alpha}$-intensity in (a) corresponds to the non-averaged $D_{\alpha}$-activity in figure 5 . The onset of the rise of the $D_{\alpha}$-intensity marking the beginning of the ELM is indicated by vertical lines. At the times $t_{E L M}$ the $D_{\alpha}$-intensity has maxima during the ELMs. The fluctuation amplitudes are reduced for about $2.5 \mathrm{~ms}$ after the ELM onset. The increased fluctuation amplitudes $\tilde{n}_{e}$ and $\tilde{T}_{e}$ for times $t-t_{E L M} \approx 2.5-5.7 \mathrm{~ms}$ are mainly due to large scale structures with $n_{\text {tor }} \leq 10$, which are not correlated with an increase in $D_{\alpha}$-intensity (b). The relative errors of the data points are $\delta\left(\tilde{n}_{e}\right) / \tilde{n}_{e} \leq 10 \%, \delta\left(\tilde{T}_{e}\right) / \tilde{T}_{e} \leq 10 \%$ and $\delta(t-$ $\left.t_{E L M}\right)=1 \mu s$. 
tuation amplitudes and quasi-mode numbers are investigated for different profile gradients in the following.

Typically used parameters describing the steep gradient region at the plasma edge are the pressure gradient length, or the pedestal width. Here we determine these parameters for the electron pressure and density profiles. The local electron pressure gradient length $l_{p e}=p_{e} /\left(d p_{e} / d R\right)$ is determined for each evaluated time point from the electron pressure $p_{e}$, and its gradient $d p_{e} / d R$ with the electron density and temperature data measured by Thomson scattering. The local gradients of electron density and temperature, $d n_{e} / d R$ and $d T_{e} / d R$, were determined over a radial width $d R \approx 1 \mathrm{~cm}$ in the poloidally averaged 1D radial profiles. The pedestal width of the electron density profile, $d_{n e}$, is estimated by $d_{n e}=\left\langle n_{e}\right\rangle_{H 5} /\left(d n_{e} / d R\right) .\left\langle n_{e}\right\rangle_{H 5}$ is a line integral of the electron density at the plasma edge including the pedestal shoulder (see figure 1) and divided by the integration path length. Both the pressure gradient length $l_{p e}$ and the pedestal width $d_{n e}$ are evaluated for single time points when the background profiles are perturbed by large scale fluctuations. Thus variations in both the pressure gradient length $l_{p e}$ and the pedestal width $d_{n e}$ in inter-ELM phases are found which are at least as large as the differences in gradient lengths between typical unperturbed pre-ELM and crashed profiles during the ELM (figures 7(a) and (b)). The strong variation of the gradient lengths and pedestal widths correlate with a variation in the amplitudes of the large scale fluctuations: The relative amplitudes of the fluctuations, $\tilde{n}_{e} /\left\langle n_{e}\right\rangle_{p o l}$ and $\tilde{T}_{e} /\left\langle T_{e}\right\rangle_{p o l}$ for the electron density and temperature respectively, are plotted versus the electron pressure gradient length $l_{p e}$ in figures $7(\mathrm{c})$ and (e), and versus the pedestal width of the electron density $d_{n e}$ in figures $7(d)$ and (f). The trend of the data in figures $7(\mathrm{c})$ - (f) shows that with smaller electron pressure gradient length $l_{p e}$, or smaller electron density pedestal width $d_{n e}$ the relative fluctuation amplitudes of both the electron density, and temperature are increasing.

The relative fluctuation amplitudes of the electron density and temperature, $\tilde{n}_{e} /\left\langle n_{e}\right\rangle_{\text {pol }}$ and $\tilde{T}_{e} /\left\langle T_{e}\right\rangle_{\text {pol }}$, are plotted versus the toroidal quasi-mode number $n_{\text {tor }}$ in figure 8 . The largest relative fluctuation amplitudes both in electron density, and temperature are found for the smallest toroidal mode numbers $n_{t o r}$. So on average with the spatial scale of the mode also its relative amplitude is increasing.

The radial profiles of the relative fluctuation amplitudes of the electron density and temperature, $\tilde{n}_{e} /\left\langle n_{e}\right\rangle_{\text {pol }}$ and $\tilde{T}_{e} /\left\langle T_{e}\right\rangle_{\text {pol }}$, are shown in figure 9 . For the time points excluding the ELM (figure 9(a)) the maxima of the mean relative fluctuation amplitudes are located in the steep gradient region between $-1.5 \mathrm{~cm} \leq R-R_{\text {out }} \leq$ $-0.5 \mathrm{~cm}$. The relative fluctuation amplitudes decrease towards the pedestal shoulder, and towards the separatrix. It is interesting to note that also relative fluctuation amplitudes up to $100 \%$ are found both for the electron density and temperature in the steep gradient region (see figure 9). These strong modulations appear then as 'holes' in the electron density and temperature with nearly zero density and temperature, as described earlier [4]. The relative fluctuation amplitudes of electron density and temperature, $\tilde{n}_{e} /\left\langle n_{e}\right\rangle_{p o l}$ and $\tilde{T}_{e} /\left\langle T_{e}\right\rangle_{p o l}$ for time points including the ELM (figure 9(b)) show the same radial profile towards the pedestal shoulder, as for the inter-ELM phases (figure 9(a)). Towards the separatrix and in the SOL the 
\# 20414 - 20
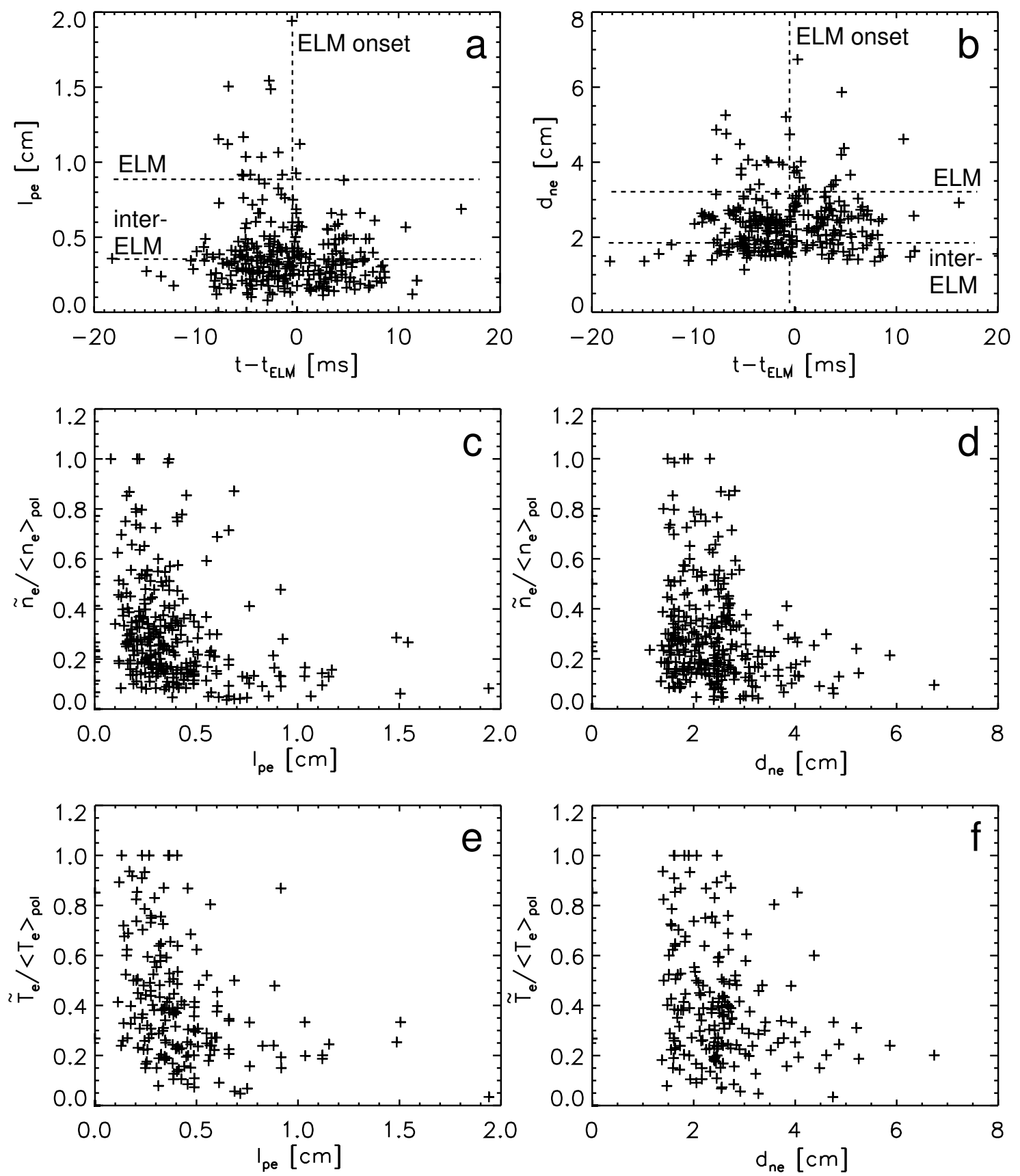

Figure 7: Plots of the time evolution of the electron pressure gradient length $l_{p e}(a)$ and electron density profile pedestal width $d_{n e}(b)$, and of $\tilde{n}_{e} /\left\langle n_{e}\right\rangle_{\text {pol }}$ and $\tilde{T}_{e} /\left\langle T_{e}\right\rangle_{p o l}$ versus $l_{p e}(c),(e)$ and $d_{n e}(d),(f)$ respectively. The typical variations of $l_{p e}$ and $d_{n e}$ between ELM and inter-ELM phases are indicated in figures (a) - (b). Note the small variation of $l_{p e}$ and $d_{n e}$ during the $E L M$, for $1 \mathrm{~ms} \leq t-t_{E L M} \leq 2 \mathrm{~ms}$, after the profile crash. The errors of the data points are $\delta\left(\tilde{n}_{e} /\left\langle n_{e}\right\rangle_{\text {pol }}\right) \leq 14 \%, \delta\left(\tilde{T}_{e} /\left\langle T_{e}\right\rangle_{\text {pol }}\right) \leq 15 \%$ and $\delta\left(l_{p e}\right) / l_{p e} \leq 14 \%, \delta\left(d_{n e}\right) / d_{n e} \leq 7 \%$. 

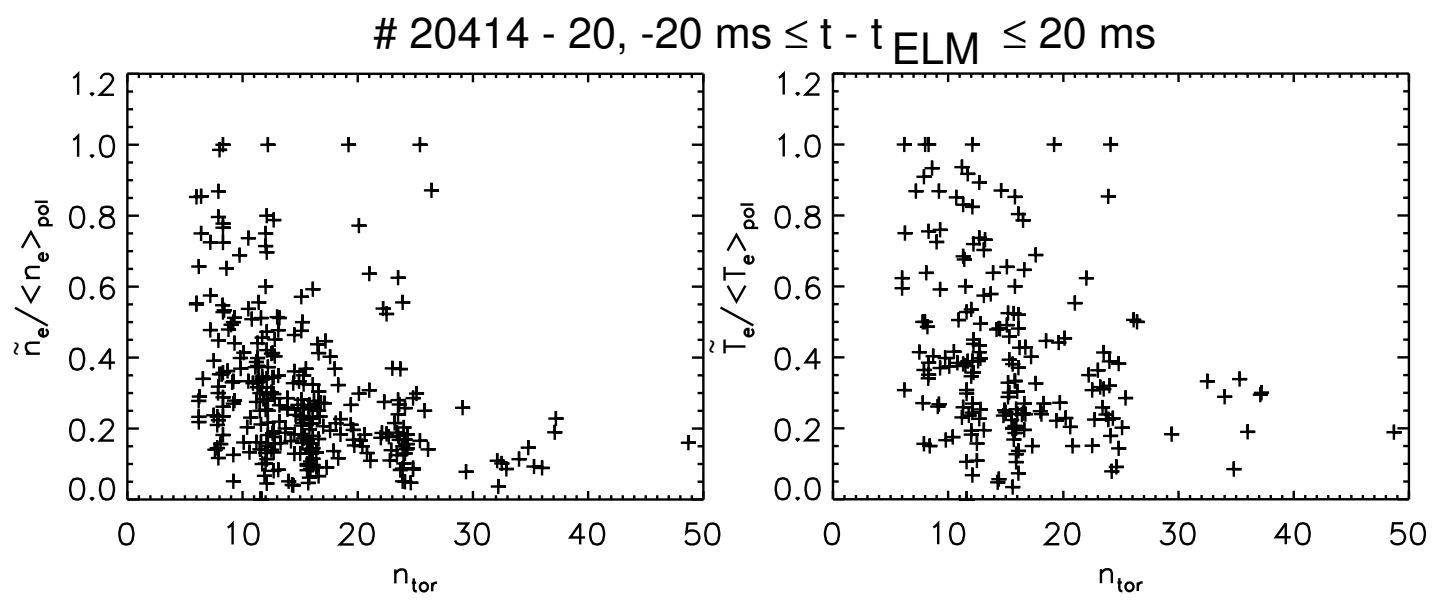

Figure 8: Plots of $\tilde{n}_{e} /\left\langle n_{e}\right\rangle_{\text {pol }}$ and of $\tilde{T}_{e} /\left\langle T_{e}\right\rangle_{\text {pol }}$ versus $n_{\text {tor }}$. The errors of the data points are $\delta\left(\tilde{n}_{e} /\left\langle n_{e}\right\rangle_{\text {pol }}\right) \leq 14 \%, \delta\left(\tilde{T}_{e} /\left\langle T_{e}\right\rangle_{\text {pol }}\right) \leq 15 \%$ and $\delta n_{\text {tor }} / n_{\text {tor }} \leq 20 \%$.

relative fluctuation amplitudes, however, are larger than in the inter-ELM phases.

\subsection{Histograms of the large scale fluctuations in $1 \mathrm{D}$ radial $H$ mode profiles of electron density and temperature}

In the previous section only quasi-periodic fluctuations with relative amplitudes above about $5 \%$ were analysed. This was possible for a fraction of $61 \%$ of all measured time points. We now look at the perturbation of the $1 \mathrm{D}$ radial profiles by all inter-ELM fluctuations. These include additionally small amplitude quasiperiodic, and non-periodic fluctuations.

1D radial profiles of electron density and temperature at the plasma edge excluding the ELM are shown in figure 10 and figure 11 respectively. The histograms $h$ of the electron densities and temperatures, which are normalized to the arithmetic means $\left\langle n_{e}\right\rangle$ and $\left\langle T_{e}\right\rangle$, are plotted for several radial positions. The arithmetic mean values $\left\langle n_{e}\right\rangle$ and $\left\langle T_{e}\right\rangle$ were determined for each scattering volume within the investigated radial range individually over a time interval where the plasma parameters were constant. Thus systematic calibration errors do not appear as fluctuations in the histograms. The intervals of the histograms have sizes of twice the mean statistical relative errors of measurement, $\delta n_{e} / n_{e}$ and $\delta T_{e} / T_{e}$. The scatter of the data, indicated by $\operatorname{Var}^{1 / 2}$ with $V$ ar as the variance of the normalized electron densities, or temperatures, is wider than the statistical errors of measurement (see figures 10 and 11). Thus mainly plasma fluctuations broaden the histograms. The symmetry, or asymmetry of the histograms can be quantified by the skewness $\gamma_{1}=\mu_{3} / \operatorname{Var}^{3 / 2}$ with $\mu_{3}$ as the third central moment of the normalized electron densities, or temperatures. Symmetric histograms (skewness $\gamma_{1} \approx 0$ ) for the electron density and temperature are found in the middle of the steep gradient region. Here the effect of maxima and minima, which perturb the background profiles, is equally strong. Further inwards the histograms are asymmetric and the skewness $\gamma_{1}$ is negative: The background profiles are on average more perturbed by the minima, or 'dips', than 


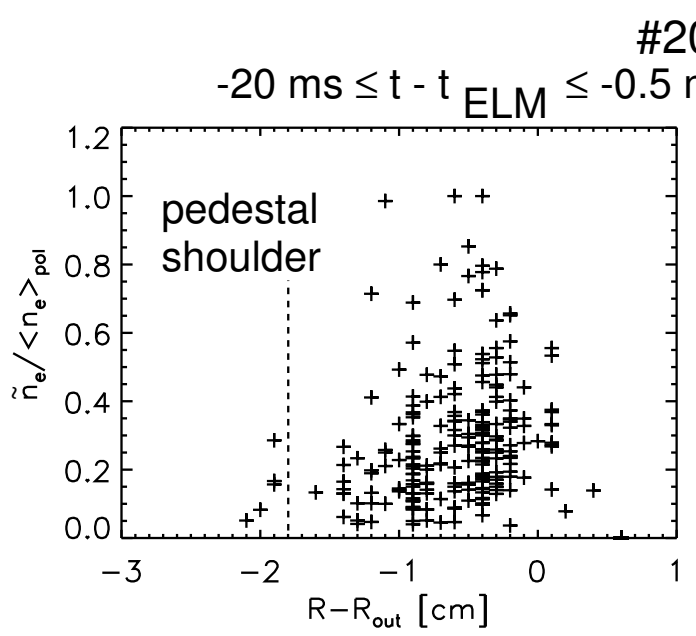

\#20414-20
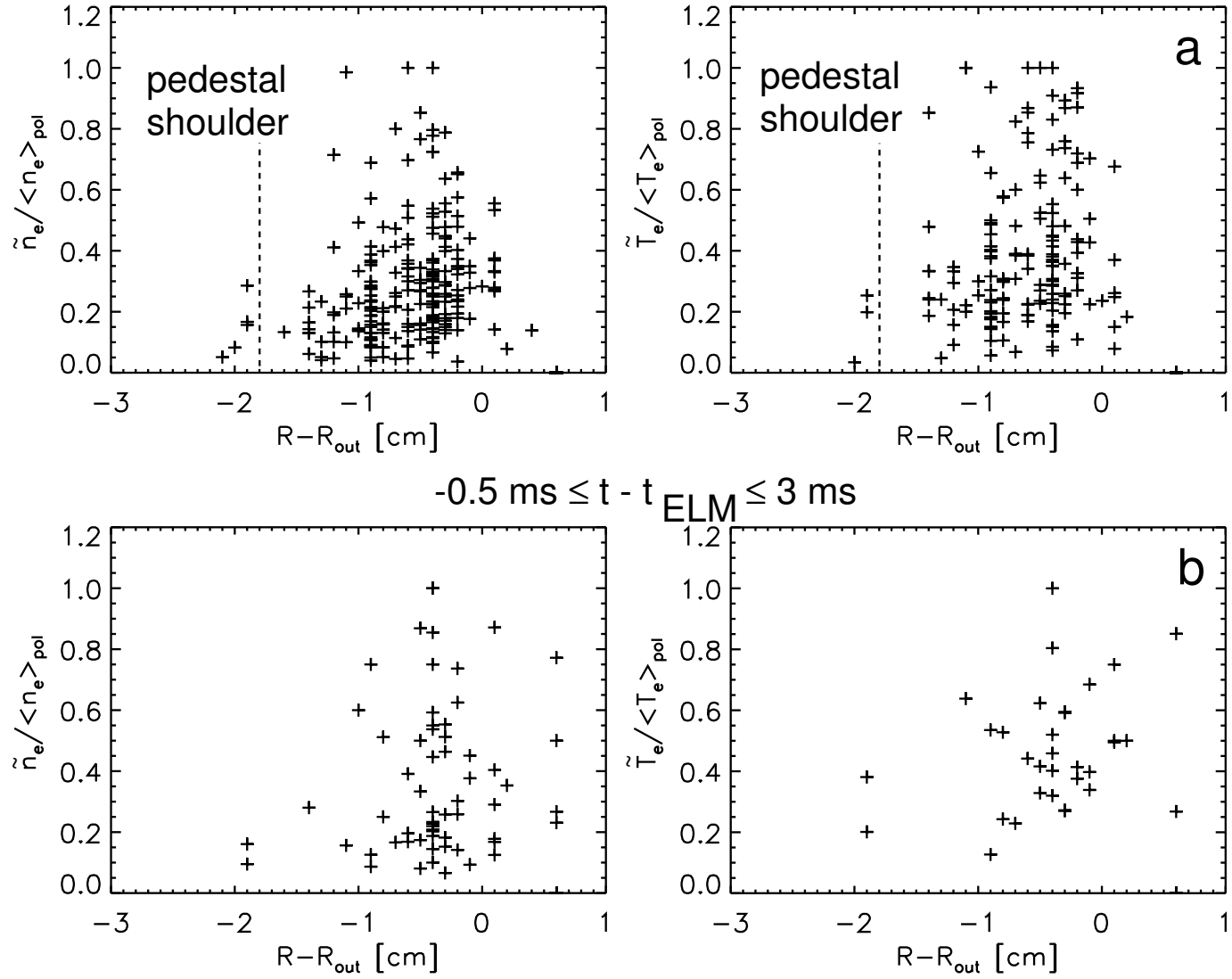

Figure 9: Radial profiles of $\tilde{n}_{e} /\left\langle n_{e}\right\rangle_{\text {pol }}$ and $\tilde{T}_{e} /\left\langle T_{e}\right\rangle_{\text {pol }}$ for inter-ELM time points (a) and time points during an ELM (b). The approximate position of the pedestal shoulder is indicated in (a). The errors of the data points are $\delta\left(\tilde{n}_{e} /\left\langle n_{e}\right\rangle_{p o l}\right) \leq 14 \%$, $\delta\left(\tilde{T}_{e} /\left\langle T_{e}\right\rangle_{\text {pol }}\right) \leq 15 \%$ and $\delta\left(R-R_{\text {out }}\right) \approx 0.5 \mathrm{~cm}$.

by the maxima, visible by the tail of the histogram at smaller values of the electron densities and temperatures. Further outwards the histograms are also asymmetric with positive skewness $\gamma_{1}$ : Here the background profile is mostly perturbed by the maxima, or 'blobs'. In the SOL the perturbations to the electron temperature are, however, again symmetric.

Because of similarities between the large scale fluctuations observed during the ELM, or in the inter-ELM phases, the histograms of figures 10 and 11 practically do not change when including the data during the ELM.

\subsection{Filamentary transport in the SOL}

The large scale fluctuations in the steep edge gradient region were analysed in the previous sections. We now look at the large scale fluctuations in the SOL. The plasmas of the discharges \#19046 - 47 were shifted to the high field side, so that with $2 \mathrm{D}$ Thomson scattering the plasma at the separatrix and in the SOL could 

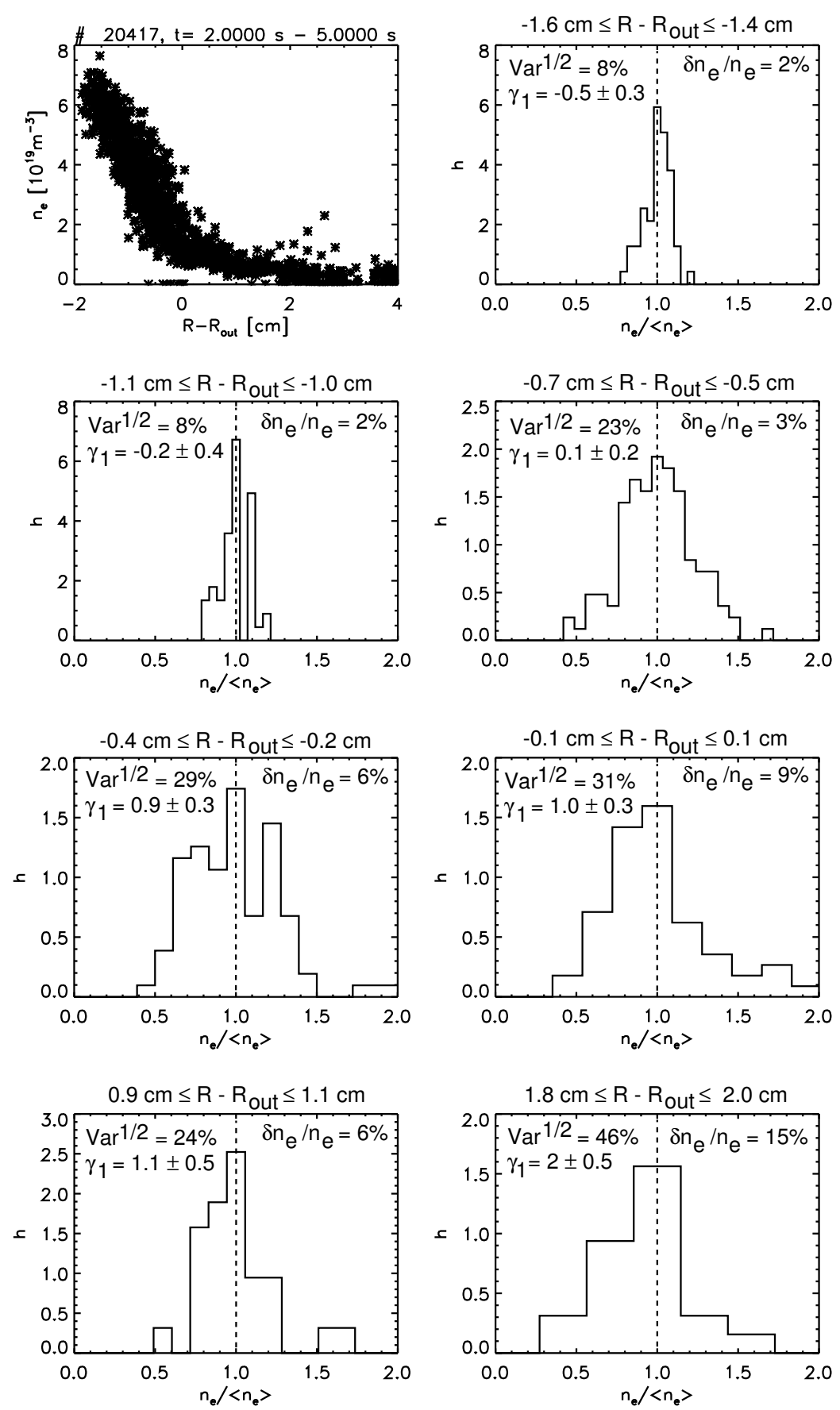

Figure 10: For $H$ mode the histograms $h$ of the electron densities $n_{e}$, normalized to the arithmetic mean $\left\langle n_{e}\right\rangle$, at different radial positions, were taken in the time interval $2 s \leq t \leq 5 \mathrm{~s}$, excluding the ELM, $t \notin\left[t_{E L M}-0.5 \mathrm{~ms}, t_{E L M}+3 \mathrm{~ms}\right]$. The intervals of the histograms have a size of twice the statistical relative errors of measurement, $\delta n_{e} / n_{e}$. The scatter of the normalized electron densities is $\operatorname{Var}^{1 / 2}$. Around $R-R_{\text {out }} \approx-0.6 \mathrm{~cm}$ the histogram is nearly symmetric (skewness $\gamma_{1} \approx 0$ ). Further inwards the background profile is more often perturbed by minima, than by maxima. Further outwards it is vice versa. 

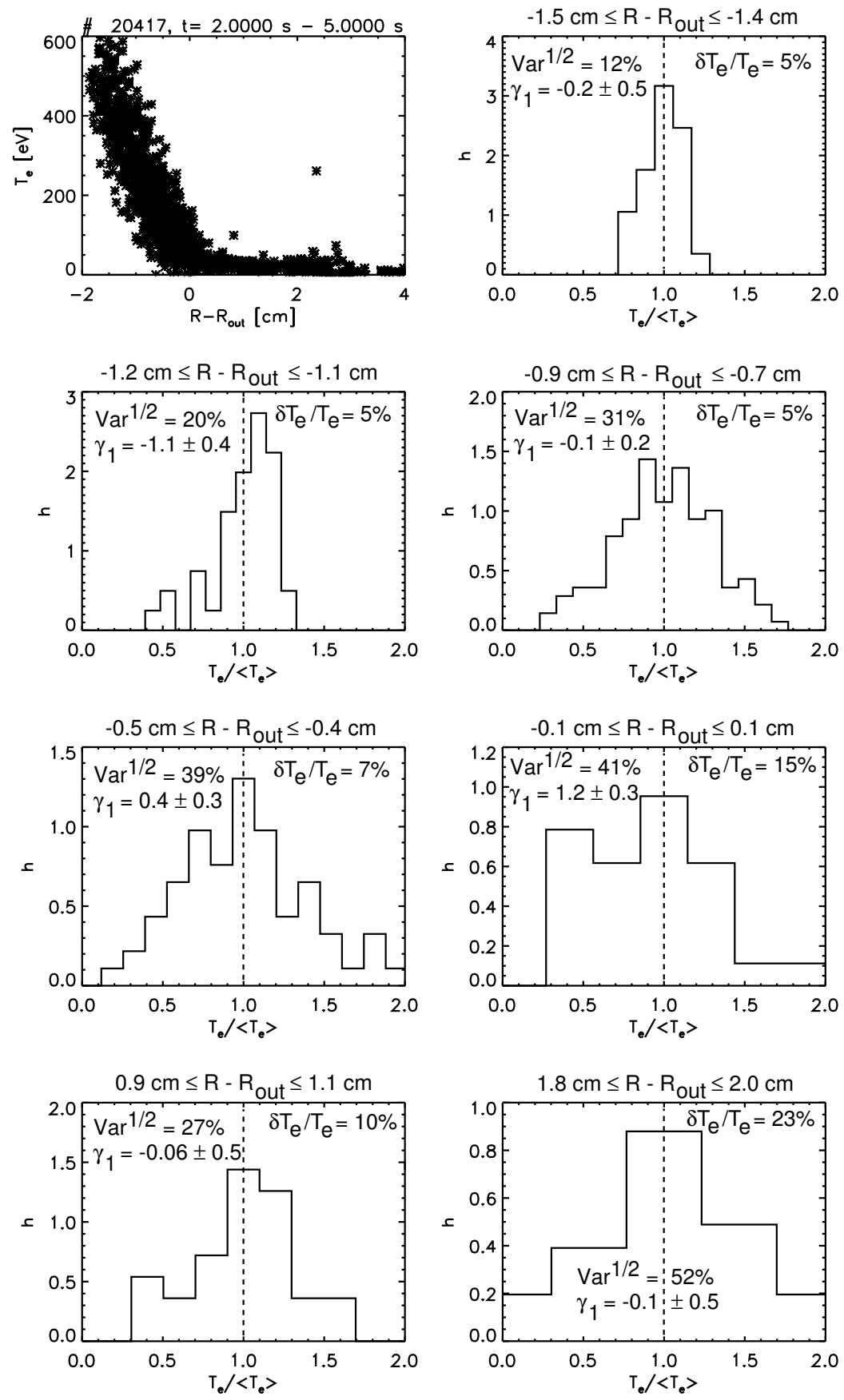

Figure 11: For $H$ mode the histograms $h$ of the electron temperatures $T_{e}$, normalized to the arithmetic mean $\left\langle T_{e}\right\rangle$, at different radial positions, were taken in the time interval $2 s \leq t \leq 5 \mathrm{~s}$, excluding the ELM, $t \notin\left[t_{E L M}-0.5 \mathrm{~ms}, t_{E L M}+3 \mathrm{~ms}\right]$. The intervals of the histograms have a size of twice the statistical relative errors of measurement, $\delta T_{e} / T_{e}$. The scatter of the normalized electron temperatures is $V a r^{1 / 2}$. Around $R-R_{\text {out }} \approx-0.8 \mathrm{~cm}$ and in the $S O L$ the histograms are nearly symmetric (skewness $\gamma_{1} \approx 0$ ). For $R-R_{\text {out }}<-0.8 \mathrm{~cm}$ the background profile is more often perturbed by minima, than by maxima. For $-0.8 \mathrm{~cm}<R-R_{\text {out }}<0.0 \mathrm{~cm}$ it is vice versa. 
be observed. For the discharge parameters see table 1. Two typical 2D plots of the electron density and temperature for an inter-ELM and an ELM phase are shown in figure 12. In an inter-ELM phase there is only one filament visible in the electron density (red circle in figure 12(a)). It has only slightly higher density than the background plasma. In a phase including the ELM 3 filaments in the electron density are observed (see the 3 circles in figure 12(b)), which have a much larger density than the background plasma. No filamentary structures are seen in the electron temperature in the SOL. Note that also symmetric histograms for the electron temperatures in the SOL are observed (figure 11). This can mean that as soon as a filament is visible in the SOL, it is no longer attached to the main plasma, but is already connected to wall structures like the divertor. Due to fast electron heat conduction the energy of the electrons is lost very quickly to first wall structures, and the electrons are cold.
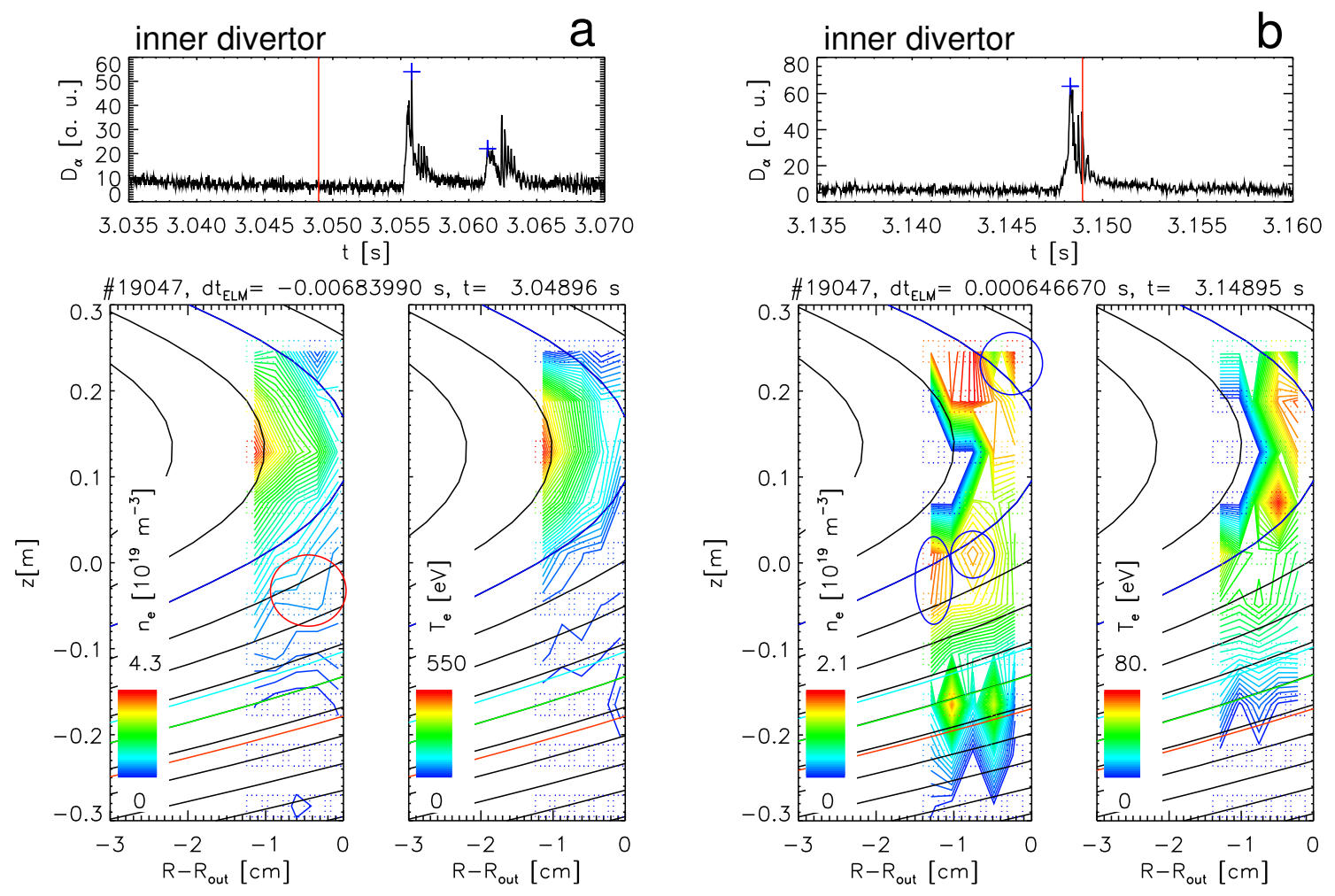

Figure 12: Filamentary transport in an inter-ELM phase (a) and during an ELM (b).

The radial velocity of the filaments can be estimated from the radial decay of the number of electrons $N_{e} / L_{\|}=n_{e, f} \times \Delta z \times \Delta R$ contained in the filaments of length $L_{\|}$, where $n_{e, f}$ is the electron density in the filament, and $\Delta z, \Delta R$ are its vertical and radial dimensions respectively. According to a simple fluid model the particles confined in a filament are lost to the divertor with the plasma sound speed $c_{s}$. When neglecting the effects due to neutral particles in this model, an exponential decay in time is expected for the number of electrons confined in a filament, $N_{e}(t)=N_{e}(0) \exp \left(-2 c_{s} t / L_{\|}\right)$. When assuming a mean radial velocity $u_{R}$ 
for all filaments, the exponential decay in time is transferred to an exponential decay in space, $N_{e}\left(R_{f}\right)=N_{e}\left(R_{\text {out }}\right) \exp \left[-\left(R_{f}-R_{\text {out }}\right) / \Delta R\right]$, with $\Delta R=L_{\|} u_{R} /\left(2 c_{s}\right)$ and $R_{f}$ as the radial position of a filament. Fits of the expression $N_{e}\left(R_{f}\right)$ to the experimental data (figure 13) are possible for decay constants $\Delta R_{i E L M}=2.2 \mathrm{~cm}$ in inter-ELM phases and $\Delta R_{E L M}=8 \mathrm{~cm}$ during the ELM. They resemble the gradient lengths of the usually plotted 1D electron density profiles for inter-ELM and ELM phases. The exponential decays found confirm the results obtained recently by Langmuir probes and Thermography in the far SOL [20]: The radial decay of both heat and particle flux is exponential with the same radial e-folding lengths. This is observed here again with Thomson scattering data, but now in the SOL near to the separatrix.

Using the plasma parameters at the separatrix and $L_{\|}=q_{95} R_{\text {out }}$ the mean velocities of the filaments, $u_{R}$, are determined from the radial e-folding lengths $\Delta R$. The mean velocity during ELMs, $u_{R, E L M}=800 \mathrm{~ms}^{-1}$, is larger than in inter-ELM phases, $u_{R, i E L M}=200 \mathrm{~ms}^{-1}$. These indirectly determined filament velocities in the SOL near the separatrix compare quite well to the radial velocity of the filaments during ELMs, $u_{R, L P}=500 \mathrm{~ms}^{-1}$, as measured by Langmuir probes in the far SOL $[20]$.

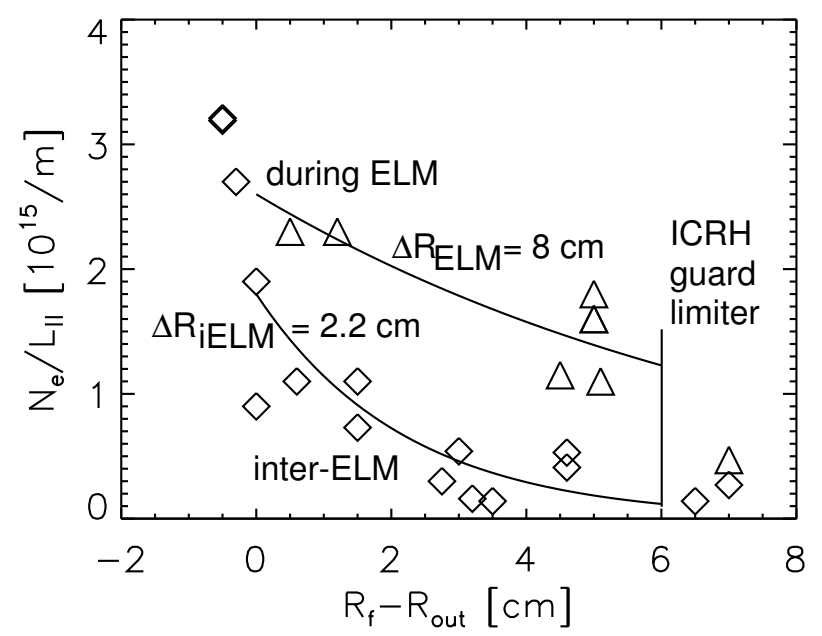

Figure 13: Plot of the number of electrons $N_{e}$ confined in the filaments of length $L_{\|}$ versus position $R_{f}$ relative to the position of the separatrix $R_{\text {out }}$ for both $E L M$, and inter-ELM phases, indicated by triangle and diamond symbols. Exponential decay functions, $N_{e}\left(R_{f}\right)=N_{e}\left(R_{\text {out }}\right) \exp \left[\left(R_{f}-R_{\text {out }}\right) / \Delta R\right]$ are fitted to the data points.

In the following we will look at the particle transport during ELMs. A number of 20 snapshots were analysed for the discharges \#19046 - 47: The resulting mean number of filaments, which are observed in a snapshot during an ELM, $\left\langle N_{f, E L M}\right\rangle=$ $2.3 \pm 0.5$, is larger than for the inter-ELM phase, $\left\langle N_{f, i E L M}\right\rangle=0.9 \pm 0.3$. The additional loss of electrons during an ELM can be estimated from the radial flux, $\Delta N_{e, E L M} \approx 3\left\langle N_{f, E L M}\right\rangle u_{R, E L M} N_{e}\left(R_{\text {out }}\right) \Delta t_{E L M} / \Delta R_{T S}$. The duration of the ELM, as seen in the $D_{\alpha}$-light in the divertor, is $\Delta t_{E L M} \approx 1 \mathrm{~ms}$, the number of electrons confined in a filament at the separatrix, is $N_{e}\left(R_{\text {out }}\right) \approx 2.5 \times 10^{15} \mathrm{~m}^{-1} L_{\|}$(see figure 13), and the radial range of observation, which is covered by Thomson scattering, 
is $\Delta R_{T S}=1.1 \mathrm{~cm}$. The factor 3 is used here because the filaments exist in a wider poloidal area than is covered by Thomson scattering. With these parameters it is estimated that a fraction of $\Delta N_{e, E L M} / N_{e, p l} \approx 4 \%$ of the number of electrons in the main plasma, $N_{e, p l}$, is lost during the ELM. This fraction is in the correct order of magnitude, indicating that the particle transport during an ELM can at least to a significant part be carried by filaments. This result is in agreement with the results obtained by Langmuir probes and $H_{\alpha} / D_{\alpha}$-diagnostics, summarized in [21] and with the results obtained recently on MAST [22].

\subsection{Fluctuations in $\mathrm{L}$ mode}
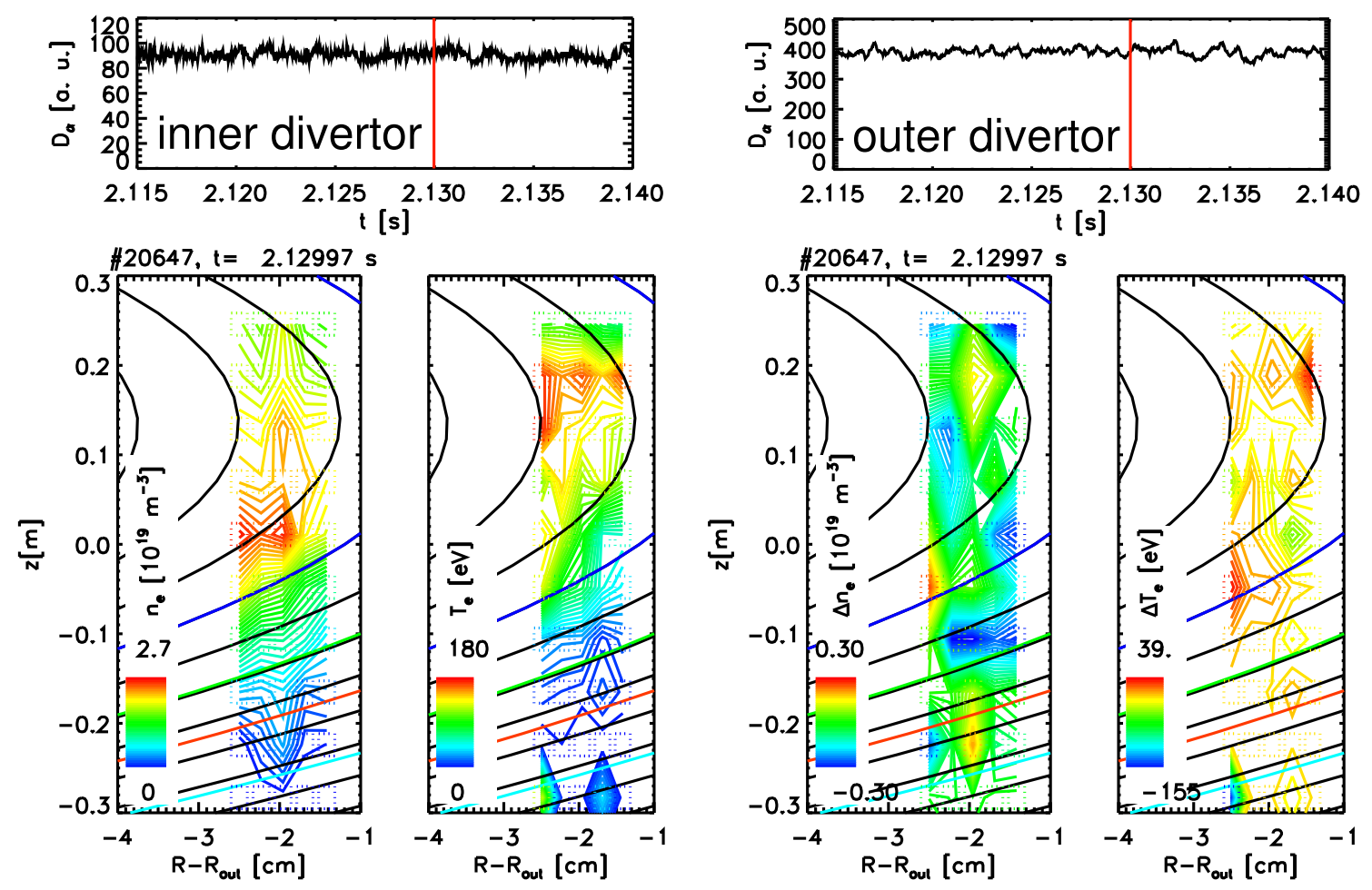

Figure 14: In L mode there are large scale, or closely packed small scale fluctuation structures. A periodicity is not clearly visible.

After the detailed analysis of the large scale fluctuations in the plasma edge of an $\mathrm{H}$ mode plasma, we now look at the fluctuations of a $\mathrm{L}$ mode plasma.

In $2 \mathrm{D}$ snapshots the $\mathrm{L}$ mode fluctuation structures, which are observed in electron density and temperature, have either a large scale, or are closely packed smaller scale structures (see figure 14). They are mainly localized in the steep edge gradient region of the pressure profile. With the limited resolution of the Thomson scattering diagnostic it was, however, not yet possible to resolve periodic structures.

Typical 1D radial electron density and temperature profiles for $\mathrm{L}$ mode show a strong scatter of the data points (see figure 15). The histograms of the electron density and temperature values, normalized to the arithmetic means $\left\langle n_{e}\right\rangle,\left\langle T_{e}\right\rangle$ were 

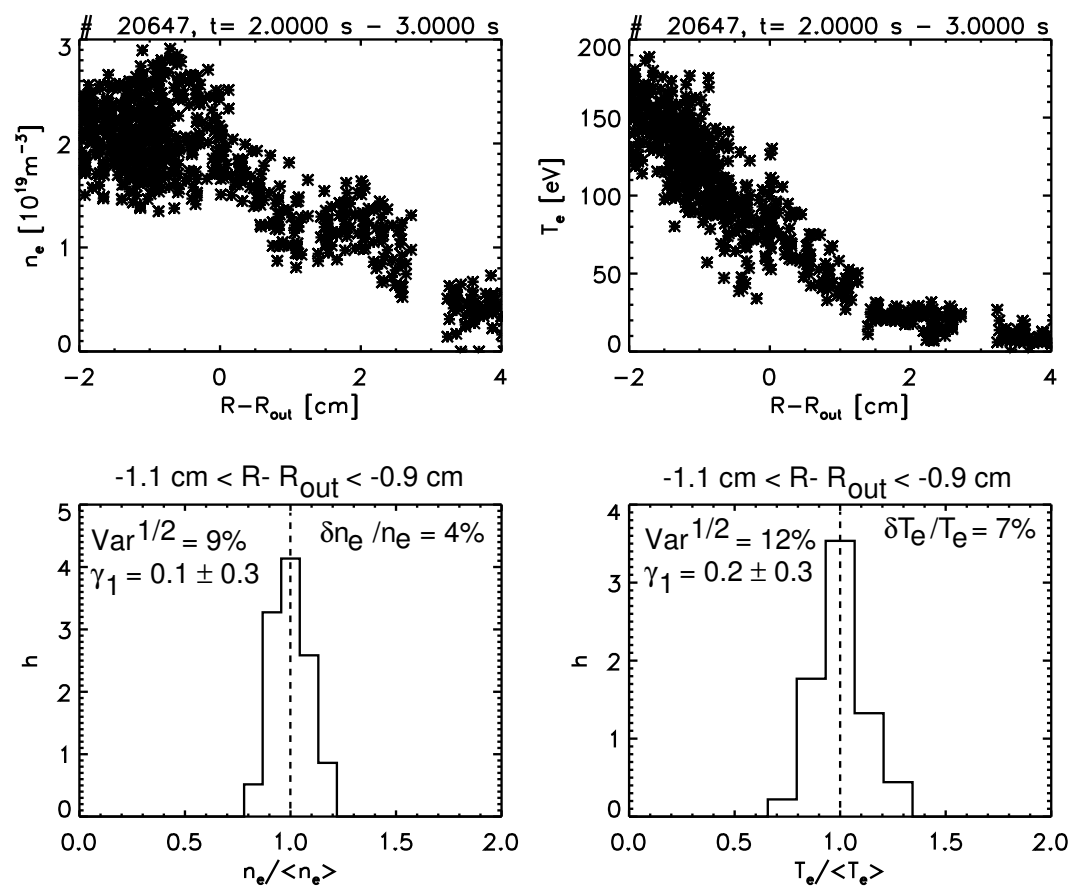

\section{steep gradient region}
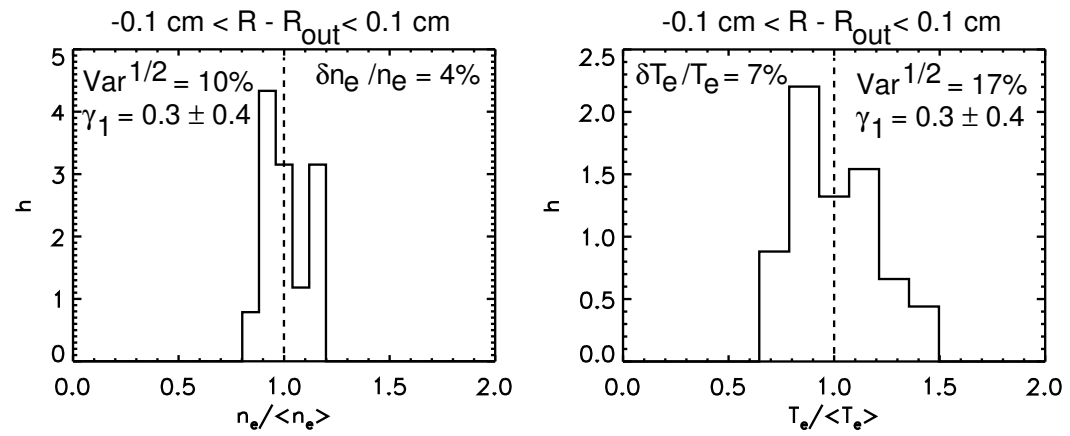

separatrix
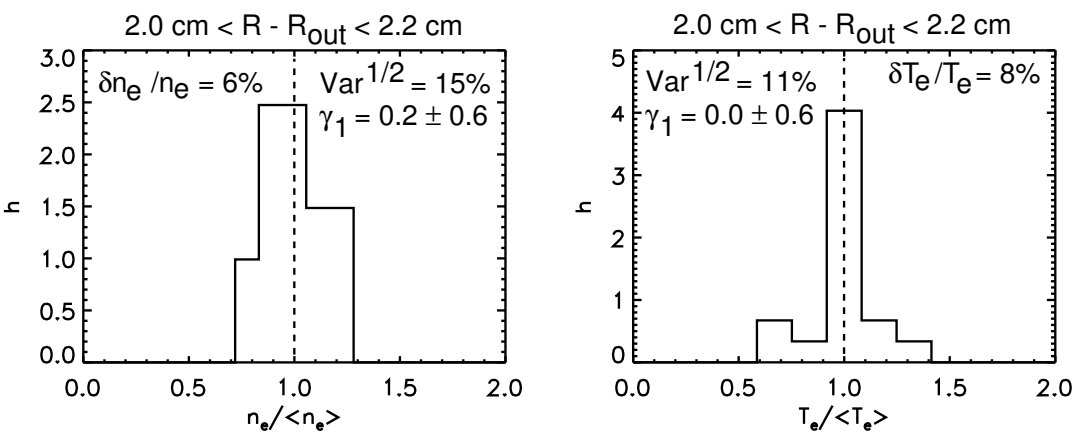

scrape-off layer

Figure 15: In $L$ mode the histograms $h$ of the electron densities $n_{e}$ (left column) and temperatures $T_{e}$ (right column), normalized to the arithmetic means $\left\langle n_{e}\right\rangle,\left\langle T_{e}\right\rangle$, were determined over the time interval $2 s \leq t \leq 3 s$, at several radial positions. The intervals of the histograms have a size of twice the statistical relative errors of measurement, $\delta n_{e} / n_{e}$ and $\delta T_{e} / T_{e}$. The scatter of the normalized electron densities and temperatures is $\operatorname{Var}^{1 / 2}$. The histograms are asymmetric with a tail towards higher values (positive skewness $\gamma_{1}$ ), indicating that on average more maxima than minima perturb the background profiles. In the SOL the histograms are symmetric $\left(\gamma_{1} \approx 0\right)$. 
determined analogously to the histograms for the $\mathrm{H}$ mode data (see section 3.4). The histograms have different shapes, depending on the radial position. The mean statistical relative errors of measurement of the electron densities and temperatures, indicated by $\delta n_{e} / n_{e}$ and $\delta T_{e} / T_{e}$ are smaller than the scatter of the data, indicated by $\operatorname{Var}^{1 / 2}$ (see figure 15). This means that mainly plasma fluctuations are responsible for broadening the distribution functions: In the gradient region inside the separatrix on average more maxima than minima perturb the background profile. This is also seen in the positive values of the skewness $\gamma_{1}$. In the SOL the histograms become more symmetric.

\section{Discussion}

The large scale fluctuations, which are observed by 2D Thomson scattering and ECE, are located in the steep gradient region of the plasma edge. Their amplitudes are increasing with decreasing local pressure gradient lengths and the electron density pedestal width. The same trend is observed for both electron density and pressure gradients, because both these quantities are correlated. There can also be correlations between the electron pressure gradient and gradients of other quantities like e. g. the gradient of the plasma current density at the edge, which was not investigated in this paper. Thus concerning the cause of these fluctuations one can only conclude that these large scale fluctuations are driven by the edge pressure gradient or by quantities correlated with this.

It was found that the amplitudes of the fluctuations in the steep gradient region are anti-correlated with the $D_{\alpha}$-intensity in the divertor. This does not necessarily mean that there is no loss of particles and heat associated with the fluctuations: For this correlation study the fluctuation amplitudes and $D_{\alpha}$-intensities were evaluated at the same time point. A time delay between a fluctuating structure in the steep gradient region and the occurrence of expelled heat and particles in the divertor is expected, because of finite parallel transport. This time delay, however, is much shorter than the time resolution of the stroboscopic Thomson scattering diagnostic of $50 \mathrm{~ms}$. No cross-correlation techniques can be applied to the Thomson scattering and $D_{\alpha}$ data to determine this time delay. The observed anti-correlation between the $D_{\alpha}$-intensity and the amplitudes of the fluctuations may be understood when considering that flat edge gradients, due to the erosion of the pedestal, are correlated with high $D_{\alpha}$-intensities.

For the occurrence of MHD modes the plasma edge should be near marginal stability. The pressure profile includes both electron, and ion pressure, of which only the electron part is known here. So there is some experimental uncertainty about the real pressure profile both before and after the ELM. If the pressure profiles before the ELM crash are marginally stable so that the ELM crash, a MHD instability, can occur, then also the pressure profiles about $3 \mathrm{~ms}$ after the ELM crash, should be marginally stable again, because here the electron density and temperature profiles are as steep as before the ELM (see figure 3). But no ELM crash occurs at this time, only large scale fluctuations are observed as described in the previous sections. 
The large scale fluctuations are seen as increased scatter in the poloidally averaged 1D radial profiles of electron density and temperature, measured by Thomson scattering. For $\mathrm{H}$ mode the positive and negative perturbations to the background profile are equally strong in the middle of the steep edge gradient region, resulting in symmetric histograms of the measured electron densities and temperatures (figures 10 and 11). Further inwards more minima and further outwards up to the separatrix more maxima perturb the background profiles. It is observed with the $2 \mathrm{D}$ snapshots of the relative electron densities and temperatures that fluctuation structures with both positive and negative perturbations are generated in the steep gradient region on the same magnetic flux surface at poloidally different positions (see figure 5). These perturbations of electron density and temperature are in phase. Thus filamentary structures with larger, or smaller pressure than the background plasma ('blobs', or 'dips') are generated. The dynamics of these filaments cannot be followed because of restrictions in the time resolution. Therefore the transport associated with these fluctuations is unclear and open questions remain: Do the fluctuations inherently have different positive and negative amplitudes, depending on the radial position of their generation? Are the large scale fluctuations mainly generated in the middle of the steep gradient region with equal positive and negative amplitudes, and the 'dips' are separated from the 'blobs' by radial drifts, leading to an accumulation of the 'dips' towards the pedestal shoulder, and of the 'blobs' towards the separatrix?

\section{Conclusion}

Large scale fluctuations are found to exist in the steep edge gradient region in interELM phases. They are observed both with Thomson scattering and ECE. The amplitudes of these fluctuations increase with decreasing electron pressure gradient length and pedestal width of the electron density profile. Thus these modes are likely driven by the edge pressure, or electron density gradient, or other gradients correlated with these quantities. In $61 \%$ of the cases these fluctuations clearly have a quasi-periodic structure with toroidal quasi-mode numbers between 6 and 48 . The relative amplitudes of these fluctuations can be up to $100 \%$. The particle loss during an ELM is at least to a significant fraction due to the electron density 'blobs' observed in the SOL.

In the poloidally averaged $1 \mathrm{D}$ radial $\mathrm{H}$ mode profiles of electron density and temperature, measured by Thomson scattering, these large scale fluctuations are the main source for the observed scatter. In the middle of the steep gradient region the scatter is symmetric with respect to the background profile, and asymmetric both further inwards (more minima), and further outwards (more maxima). This must be taken into account when determining the background profiles of electron density and temperature from the measured data.

Theoretical work is necessary to understand the nature of these fluctuations. 


\section{References}

[1] F. Wagner et al., Phys. Rev. Lett. 49, 1408 (1982)

[2] W. Suttrop, Plasma Phys. Control. Fusion 42, A1 (2000)

[3] J. Neuhauser et al. and ASDEX Upgrade Team, IAEA Fusion Energy Conf. (Chengdu, China), paper IAEA-CN-149/EX/P8-2 (2006);

J. Neuhauser et al. and ASDEX Upgrade Team, "Structure and Dynamics of Spontaneous and Induced ELMs on ASDEX Upgrade", submitted to Nucl. Fus.

[4] B. Kurzan, H. D. Murmann, J. Neuhauser and ASDEX Upgrade Team, Phys. Rev. Lett. 95, 145001 (2005)

[5] A. Herrmann, T. Eich, V. Rohde, C. J. Fuchs, J. Neuhauser and ASDEX Upgrade Team, Plasma Phys. Control. Fusion 46, 971 (2004)

[6] T. Eich et al. and ASDEX Upgrade Team, Plasma Phys. Control. Fusion 47, $815(2005)$

[7] A. Kirk et al. and ASDEX Upgrade and MAST Teams, Plasma Phys. Control. Fusion 47, 995 (2005)

[8] H. W. Müller et al. and ASDEX Upgrade Team, 29th EPS Conf. (Montreux, Switzerland), contribution O-2.06 (2002)

[9] T. Bolzonella, H. Zohm, M. Maraschek, E. Martines, S. Saarelma, S. Günter and ASDEX Upgrade Team, Plasma Phys. Control. Fusion 46, A143 (2004)

[10] I. Nunes, G. D. Conway, A. Loarte, M. Manso, F. Serra, W. Suttrop, CFN and ASDEX Upgrade Teams, Nucl. Fusion 44, 883 (2004)

[11] G. D. Conway, J. Schirmer, S. Klenge, W. Suttrop, E. Holzhauer and ASDEX Upgrade Team, Plasma Phys. Control. Fusion 46, 951 (2004)

[12] C. P. Perez et al. and JET-EFDA Contributors, Nucl. Fus. 44, 609 (2004)

[13] P. Smeulders, G. D. Conway, B. Alper, B. Balet, D. V. Bartlett, D. Borba, N. Deliyanakis, T. C. Hender and O. J. Kwon, Plasma Phys. Control. Fusion 41, 1303 (1999)

[14] C. P. Perez et al. and JET EFDA contributors, Plasma Phys. Control. Fusion 46, 61 (2004)

[15] J. A. Snipes, B. LaBombard, M. Greenwald, I. H. Hutchinson, J. Irby, Y. Lin, A. Mazurenko and M. Porkolab, Plasma Phys. Control. Fusion 43, L23 (2001)

[16] H. Murmann, S. Götsch, H. Röhr, H. Salzmann and K. H. Steuer, Rev. Sci. Instrum. 63, 4941 (1992) 
[17] B. Kurzan, M. Jakobi, H. Murmann and ASDEX Upgrade Team, Plasma Phys. Control. Fusion 46, 299 (2004)

[18] W. Schneider, P. J. McCarthy, K. Lackner, O. Gruber, K. Behler, P. Martin and R. Merkel, Fusion Eng. Des. 48, 127 (2000)

[19] W. Suttrop, A. G. Peeters, ASDEX Upgrade Team and NBI group, "Practical Limitations to Plasma Edge Electron Temperature Measurements by Radiometry of Electron Cyclotron Emission" (Garching: Max-Planck-Institut für Plasmaphysik) Report IPP 1/306 (1996)

[20] A. Herrmann, A. Kirk, A. Schmid, B. Koch, M. Laux, M. Maraschek, H. W. Müller, J. Neuhauser, V. Rohde, E. Wolfrum and ASDEX Upgrade Team, 17th PSI Conf. (Hefei, China), contribution O-34 (2006)

[21] M. Endler, I. Garcia-Cortés, C. Hidalgo, G. F. Matthews, ASDEX Team and JET Team, Plasma Phys. Control. Fusion 47, 219 (2005)

[22] A. Kirk et al. and MAST Team, Plasma Phys. Control. Fusion 48, B433 (2006) 